\title{
Nighttime Auroral Energy Deposition in the Middle Atmosphere
}

\author{
R. A. Goldberg and C. H. JaCkman \\ Laboratory for Planetary Atmospheres, NASA Goddard Space Flight Center, Greenbelt, Maryland
}

J. R. BARCUS

Department of Physics, University of Denver, Colorado

F. S $\phi$ RAAS

Department of Physics, Unversity of Bergen, Norway

\begin{abstract}
From 1976 through 1982, eight distinct nighttime auroral events have been probed with rocket payloads in a series of high lattude studies at Poker Flat Research Range, Alaska, and And $\phi y a$, Norway. The instrument packages have contained $\mathrm{X}$ ray and energetic electron detectors, permitting a measure of these energy forms and their absorption in the middle atmosphere. Although the specifics of each event show a wide range of values for energy flux and spectral hardness, certain general characteristics persist in all cases. The primary energy sources for the middle atmosphere under the active conditions examined here are mainly relativistic electrons and bremsstrahlung $X$ rays. Each of these sources is found to dominate ionization in a separate altitude region, with the electrons usually controlling ionization in the upper heights above $55-60 \mathrm{~km}$ and the $X$ rays below to about $40 \mathrm{~km}$, where cosmic rays take over. The relativistic electron ionizing radiation source has not usually been considered in modeling studies for the ion chemistry of the lower mesosphere $(55-70 \mathrm{~km})$, yet this radiation is capable of increasing ion concentration and electrical conductivity in excess of a factor of 10 . Moreover, the persistence of relativistic electrons as an important source in all events studied here strongly suggests that this deficiency be corrected.
\end{abstract}

\section{INTRODUCTION}

Recent interest in high-latitude energetic particles and their effect on the middle atmosphere has centered on the sensitivity of ozone to such radiations. This has largely been stimulated by the findings of Heath et al. [1977], who measured an ozone depletion in the stratosphere during the great solar proton event (SPE) of August 1972 using the backscatter ultraviolet (BUV) instrument on Nimbus 4. Earlier, Weeks et al. [1972] suggested such an effect from rocket data, but the satellite data were essential to demonstrate the global magnitude, extent, and temporal response of atmospheric ozone to the solar protons. Since that result, numerous theoretical papers have appeared calculating and modeling atmospheric response to the August 1972 event [e.g., Reagan et al., 1981; Solomon and Crutzen, 1981] and other SPE's [e.g., Swider and Keneshea, 1973; Crutzen et al., 1975; Frederick, 1976; McPeters et al., 1981; Solomon et al., 1983]. Simultaneously, Thorne [1977, 1980] has suggested that relativistic electron precipitation (REP) events, although weaker than SPE's in terms of atmospheric penetration and energy flux, could significantly contribute to ozone modification because of their higher frequency of occurrence. Thorne's estimates of REP frequency and spectral structure are based on ground-based and balloon measurements [e.g., Bailey and Pomerantz, 1965; Bailey, 1968; Rosenberg et al., 1972; Larsen and Thomas, 1974; Thorne and Larsen, 1976] and satellite data [Vampola, 1971; Reagan, 1977; Thorne, 1978], all of which provide long-term sampling but have limitations with respect to direct observation of the complete energy spectral range, in situ atmospheric deposition

This paper is not subject to U.S. copyright. Published in 1984 by the American Geophysical Union.

Paper number 4A0493. characteristics, or simply being in the right place at the right time. The 1971-089A satellite data [Larsen et al., 1976] are used to derive ion pair production rates by relativistic electrons during disturbed conditions over Ottawa. Using models, these data show the dominance of ion pair production rates by electrons at heights between about 55 and $89 \mathrm{~km}$ over those due to solar radiation and galactic cosmic rays. Most recently, Vampola and Gorney [1983] have also modeled the importance of REP events on the middle atmosphere using data from the S3-2 satellite.

In this work we report on our findings concerning relativistic electron showers determined from nighttime measurements with rockets during eight distinct aurorally active events at Poker Flat Research Range, Alaska, and And $\phi$ ya, Norway. These nighttime measurements were made from 1976 to 1982. Energetic electron fluxes were found to occur in all events studied, but with magnitudes inadequate to accommodate significant changes in stratospheric ozone. On the other hand, the fluxes provided dominant ionizing radiation in the lower mesosphere, making them an important element of middle atmospheric electrodynamics in terms of ion conductivity, mobility, electric field structure, and any models which might consider ion-neutral chemistry for this region.

\section{EXPERIMENT DESCRIPTION}

Geophysical Conditions, Rocket Logistics, and Site Parameters

Table 1 shows a listing of all rockets flown to obtain the results reported here. Table 2 provides a listing of the various programs, site locations, rocket flight logistics, and other relevant background information for the data taken during magnetically active periods. We note that four separate campaigns were involved beginning with Aurorozone I in September 
TABLE 1. Rocket Summary

\begin{tabular}{lcclll}
\hline \multicolumn{1}{c}{ Program } & $\begin{array}{c}\text { Rocket } \\
\text { Number }\end{array}$ & Code* & \multicolumn{1}{c}{$\begin{array}{c}\text { Rocket } \\
\text { Type }\end{array}$} & $\begin{array}{c}\text { Magnetic } \\
\text { Condition } \\
\text { at Launch } \dagger\end{array}$ & \multicolumn{1}{c}{ Date } \\
\hline Aurorozone I & 18.178 & A & Nike Tomahawk & disturbed & Sept. 21, 1976 \\
& 18.179 & A & Nike Tomahawk & disturbed & Sept. 23, 1976 \\
Aurorozone II & 15.165 & B & Super Arcas & quiet & March 22, 1978 \\
& 15.168 & B & Super Arcas & disturbed & March 29, 1978 \\
MAE I & 15.169 & B & Super Arcas & disturbed & March 27, 1978 \\
& 30.010 & C & Orion & disturbed & Oct. 7, 1980 \\
& 30.011 & C & Orion & quiet & Oct. 11, 1980 \\
& 33.015 & D & Honest John Orion & disturbed & Oct. 7, 1980 \\
& 33.016 & D & Honest John Orion & quiet & Oct. 11, 1980 \\
MAE II & 33.017 & D & Honest John Orion & disturbed & Oct. 18, 1980 \\
& 31.024 & E & Nike Orion & disturbed & Jan. 24, 1982 \\
& 31.025 & E & Nike Orion & disturbed & Jan. 23, 1982 \\
\hline
\end{tabular}

*For use in Table 3.

$\dagger$ See text.

1976 and followed by Aurorozone II in March 1978, Middle Atmospheric Electrodynamics (MAE) I in October 1980, and MAE II in January 1982. The corresponding riometer records for active nighttime periods during which rocket launches occurred are presented in Figures 1-4. For Aurorozone I the records are provided for both College $(50 \mathrm{~km}$ southwest of Poker Flat) and Fort Yukon ( $200 \mathrm{~km}$ downrange), Alaska, to bracket the region where the primary data were acquired. For all other cases, riometer records are provided for stations at the launch sites, since these rocket flights acquired data within a region of $50 \mathrm{~km}$ or less to the station.

Furthermore, for all launches conducted at Poker Flat Research Range, Alaska, the nearby Chatanika Radar Observatory (CRO) provided coordinated data to help predict and then define the conditions for each launch. Figure 5 [after Goldberg, 1979] shows an example of the CRO measured electron density profiles for the two launches during Aurorozone I. For the MAE I launches in And $\phi y a$, real time confirmation of the areal extent for the event in progress was made with the partial reflection experiment in Troms $\phi$.

From these and other data not shown, the following conditions were in effect for the measurements discussed in this work. For Aurorozone I, NASA 18.178 was launched into a modest auroral breakup which was most intense above the launch site. NASA 18.179 made measurements during the similar period for an event of much greater extent to the north, and with significantly higher riometer absorption at both College and Fort Yukon. Both of these events are discussed in detail in the work of Barcus et al. [1981]. During Aurorozone II, NASA 15.169 was launched into a breakup exhibiting the highest riometer absorption of any event measured in this work. A description of this event was also made simultaneously with a high-altitude overhead flight (NASA 18.215) using a scanning $X$ ray detector to build up images of the auroral structure [Goldberg et al., 1982]. NASA 15.168 was launched 2 nights later during weak ionospheric absorption but at a time when CRO was indicating higher than normal fluxes of energetic electrons. A third rocket, NASA 15.165 , was launched into relatively quiet conditions to obtain baseline data for comparison with the active periods.

MAE I was conducted in And $\phi y a$, Norway. For this program, rocket pair sequences were launched during both quiet and disturbed conditions. NASA 30.010 and 33.015 were launched $1 \mathrm{~min}$ apart to gain simultaneous data during the recovery phase of a sustained but weak absorption event. NASA 33.017 was launched 11 nights later during an extended period of pulsating aurora. Finally, two near-simultaneous flights, NASA 33.016 and 30.011 , were launched during a quiet night to once again make baseline measurements and for electrodynamic studies of the middle atmosphere reserved for discussion elsewhere. Because And $\phi y a$ is situated at a somewhat greater magnetic latitude than Poker Flat within the auroral oval, we anticipated and were able to verify (see discussion later in this work) that the characteristic energetic electron and $X$ ray fluxes would contain softer spectra than those observed at Poker Flat. This combined with observed weaker intensities accounts for the typically lower riometer absorption seen as a general characteristic of the events we studied in And $\phi y a$.

For MAE II we returned to the more energetic environment of Poker Flat, since our prime interest related to the impact of the measured radiation on middle atmospheric electrical structure. Both launches here (NASA 31.024 and 31.025) were made into postbreakup conditions during which diffuse aurorae covered nearly the entire visible sky. For 31.025 the aurorae were imbedded with pulsations which included some of about 2-min duration, permitting our study of energy enhancements induced by temporal variations. During the flight of 31.024, no pulsations were observed, and the riometer records exhibited weak absorption $(0.7 \mathrm{~dB}$; see Figure $4 b)$. Moreover, the measured energy distribution was found to be dominated by relativistic electrons in a manner resembling the much stronger event during Aurorozone II (15.168).

\section{Instrumentation}

All payloads fit into a category called the XRG grouping [Goldberg, 1979] except for five payloads which we shall refer to as high fliers (18.178 and 18.179 in Aurorozone $I$ and 33.015-33.017 in MAE I). That portion of the basic instrumentation used in this work and common to all versions of the $X R G$ payload consisted of a zenith-viewing $X$ ray detector, a Geiger tube guard counter, and a two-axis magnetometer to estimate payload aspect and stability. On the XRG flights in the MAE I program, a solid state detector was added to measure relativistic electrons. For all XRG payloads, prime data acquisition occurred during the parachute descent portion of each flight. The high fliers, 18.178 and 18.179 , carried $X$ ray detectors and arrays of Geiger tubes, whereas 33.015-33.017 


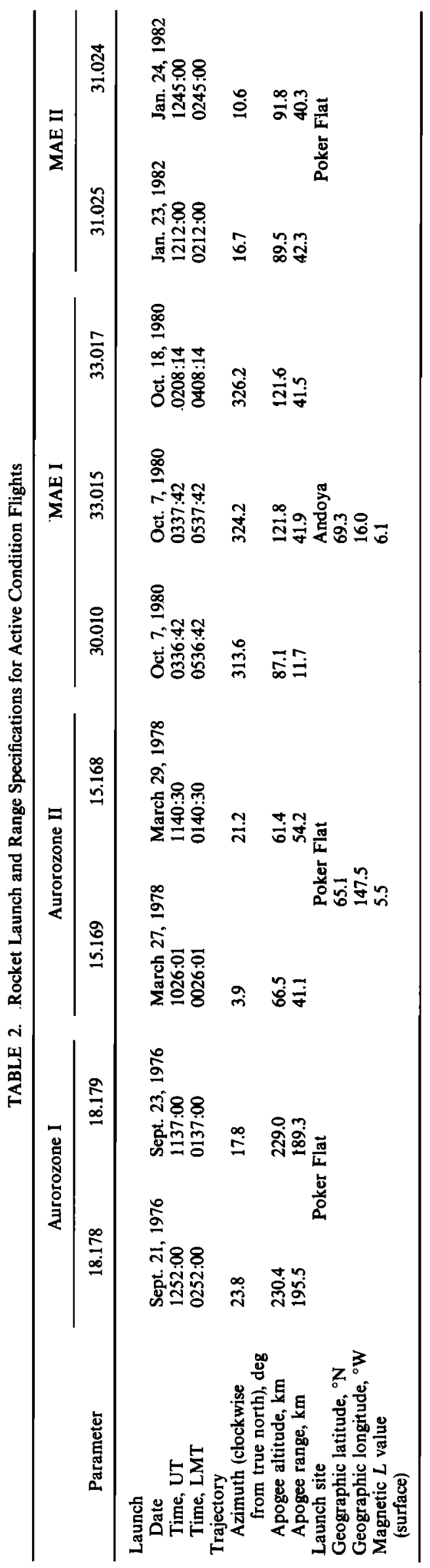

a
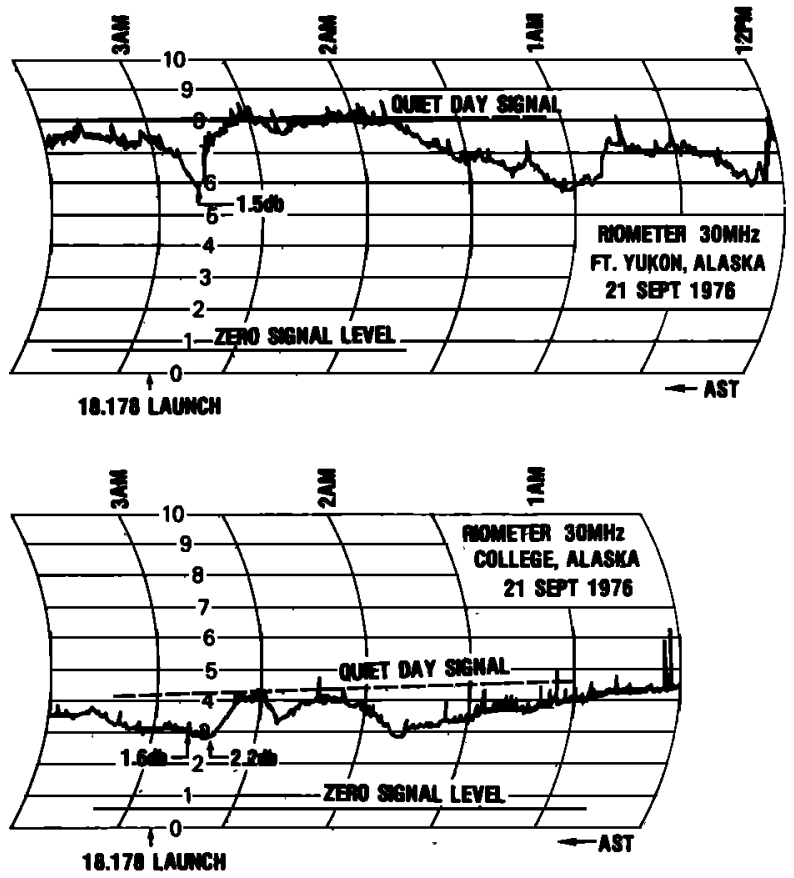

18.170 LACMCH

b
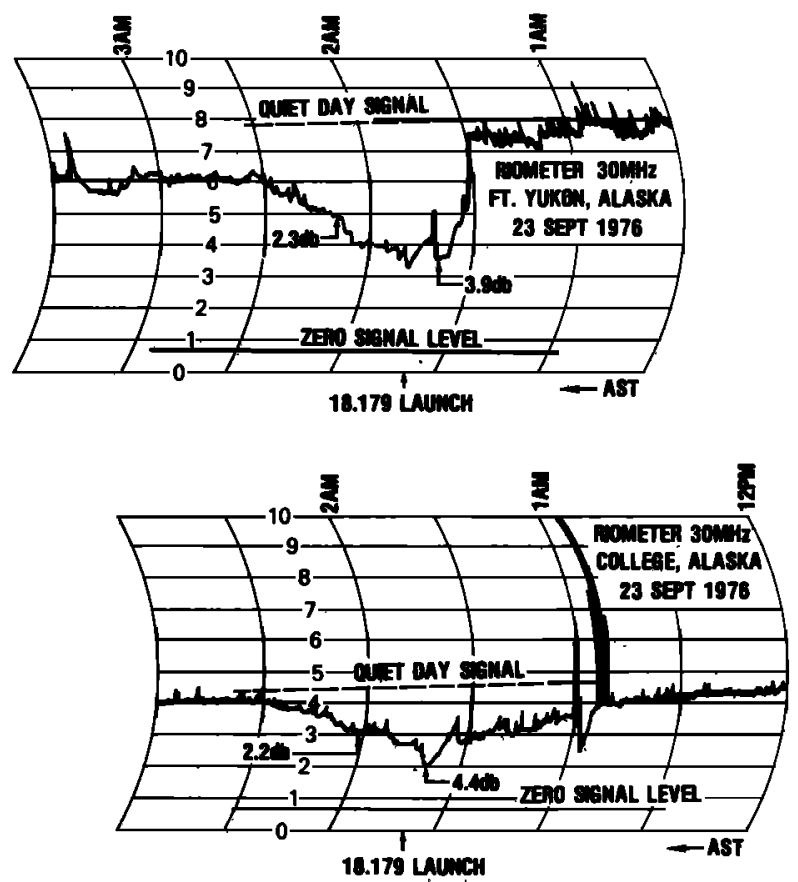

Fig. 1. Comparison of riometer absorption records for College (50 km southwest), and Fort Yukon ( $200 \mathrm{~km}$ north), Alaska, with the indicated launch times (AST) for $(a) 18.178$ and (b) 18.179 during Aurorozone I.

carried a complement of instruments similar to the XRG's with solid state detectors. Table 3 summarizes the characteristics of essential instrumentation for each flight.

All $X$ ray measurements were made using $\mathrm{NaI}$ scintillation crystals fitted with a thin Be entrance window and optically mounted to a ruggedized photomultiplier. The detectors used 

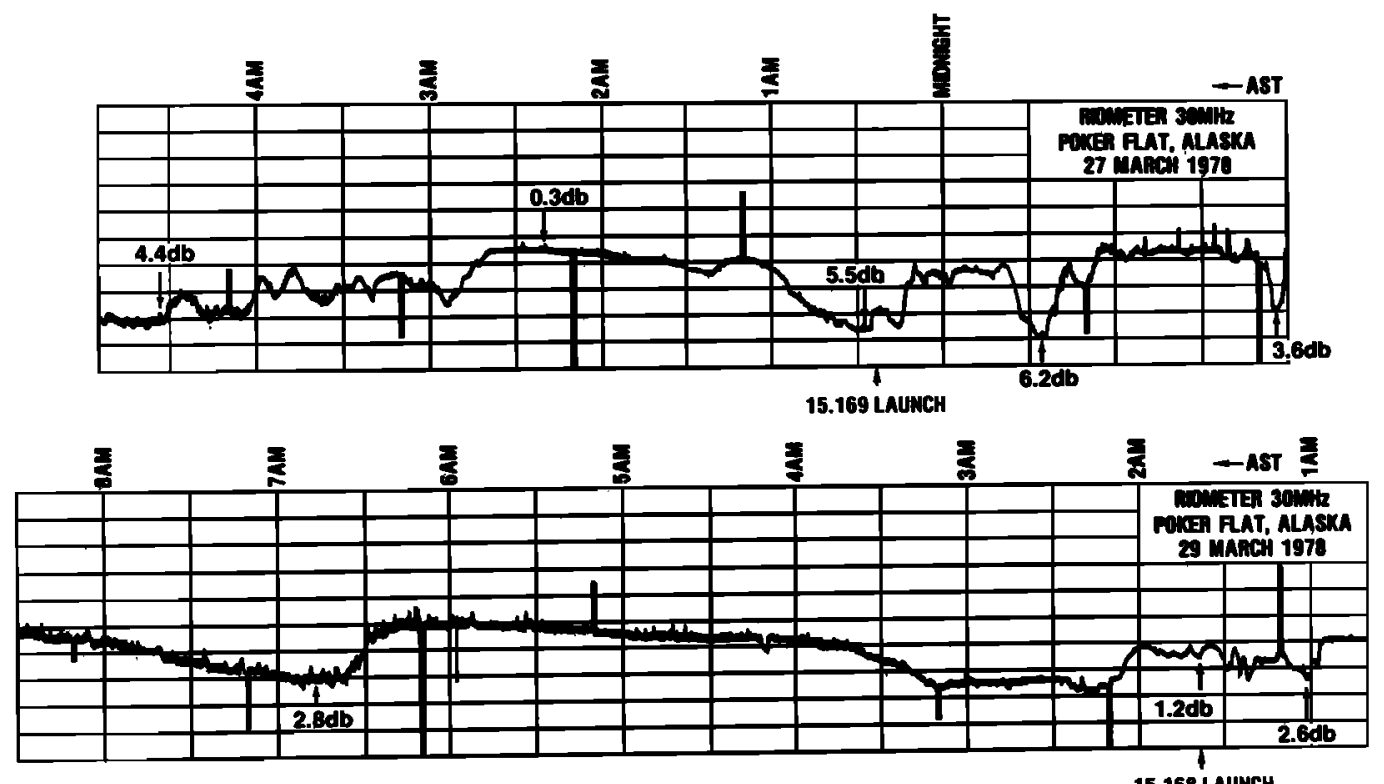

15.168 LAUNCH

Fig. 2. Riometer absorption records for Poker Flat, Alaska, during Aurorozone II with the indicated launch times (AST) for 15.169 and 15.168 .

have high efficiency for $X$ rays of moderate energy down to about $5 \mathrm{keV}$. Electronic sampling of the pulse amplitudes permitted spectral measurements in the energy ranges $>5,>10$, $>20$, and $>40 \mathrm{keV}$. The $X$ ray detectors used on 31.024 and 31.025 in MAE II included an additional integral channel of $>80 \mathrm{keV}$. For the high-flier $\mathrm{X}$ ray detectors the first three channels were electronically sorted in a differential instead of an integral mode. All the $\mathrm{X}$ ray detectors were also sensitive to energetic charged particles which can penetrate the Be entrance window. For electrons the threshold energy is approximately $150 \mathrm{keV}$.

Both the zenith-viewing $X$ ray detectors on the XRG payloads and the $45^{\circ}$ (relative to spin axis) detectors on the high fliers were wide angle. For the high-flier detectors, no account was made for the somewhat greater geometric factor for highenergy ( $>40 \mathrm{keV}$ ) $\mathrm{X}$ rays, which were not completely shielded by the detector collimator or the $6.35-\mathrm{mm}$-thick aluminum payload skin. The normal data acquisition period for these flights was during upleg, although for 33.015-33.017 the payloads detached and descended by parachute, permitting measurements during downleg as well.

All solid state detectors were mounted to view at $45^{\circ}$ zenith angle during parachute descent. For the high fliers they also viewed at $45^{\circ}$ to the spin axis during upleg. The XRG (30.010, $30.011)$ solid state detectors used were of the lithium-drifted silicon type ( $2 \mathrm{~mm}$ thick), sealed and ruggedized for flight. On each of the high fliers 33.015-33.017, two surface barrier silicon detectors were flown with thicknesses of $0.3 \mathrm{~mm}\left(S_{2}\right)$ and $2.0 \mathrm{~mm}\left(\mathrm{~S}_{1}\right)$. Since the noise figures for the above detectors are about $10 \mathrm{keV}$, no cryogenic cooling was necessary to maintain the coarse energy resolution used for the experiment.

Finally, all XRG payloads carried miniaturized Geiger tube detectors to measure the integral electron flux above an approximate energy threshold of $40 \mathrm{keV}$. Furthermore, 18.178 and 18.179 carried additional Geiger tubes with thresholds of 15,25 , and $100 \mathrm{keV}$ to permit measurement of the lowerenergy portion of the electron spectrum during those flights.

\section{Data Analysis}

The primary auroral energy sources measured and considered in this work are bremsstrahlung $\mathrm{X}$ rays and relativistic electrons. Because each measurement provides us with information regarding the absorption of these energy fluxes as a function of altitude, we have been able to construct height profiles of the energy deposition for each of the events studied. These are displayed in terms of ion pair production rates, based on the $34.5 \mathrm{eV} /$ ion pair suggested by Parter et al. [1976].

The details of the analyses leading to the various profiles are discussed below. The approaches used for the $\mathrm{X}$ ray and electron sources are treated separately but combined along with the calculated ion pair production rates from cosmic ray fluxes [Nicolet, 1975] to produce the total deposition curves. The cosmic ray fluxes have been adjusted for site location and phase of the solar cycle. We also note that the electron energy deposition profiles were obtained in three separate ways, depending on the instrumentation available for the measurements. For the Aurorozone I series and for MAE II, we were forced to deduce electron spectra $(E>150 \mathrm{keV})$ from the decrease in count rates with atmospheric penetration observed on the highest integral channel of the $\mathrm{X}$ ray detectors. This decrease occurred well above those altitudes expected for $X$ ray absorption of these energies. Thus owing to the sensitivity of the detector to energetic electrons in excess of $150 \mathrm{keV}$, we deduced that the decay in count rates must be caused by absorption of energetic electrons in the atmosphere. The shape of the absorption curve allowed construction of the electron spectrum from range-energy considerations. For the Super Arcas flights in Aurorozone II, this spectrum was constructed using direct electron data from Geiger tube counters with a 40-keV threshold, providing us with a more accurate spectrum. Both of these procedures assume that during the period of descent through the region of electron absorption, the source remains nearly constant in time, which appeared to be the case based on other supporting measurements. 


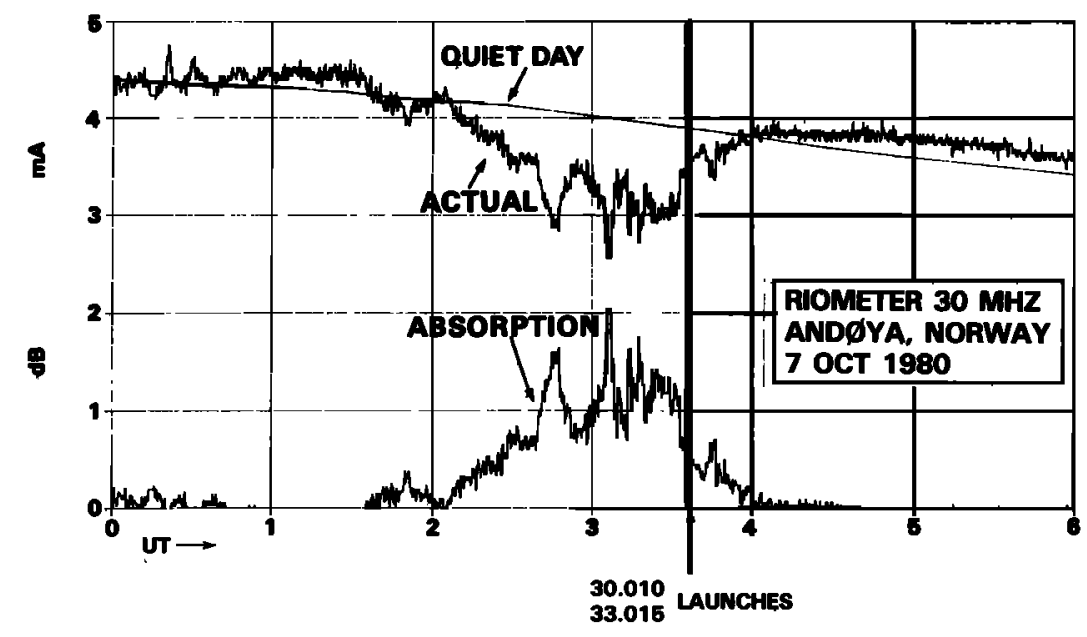

b

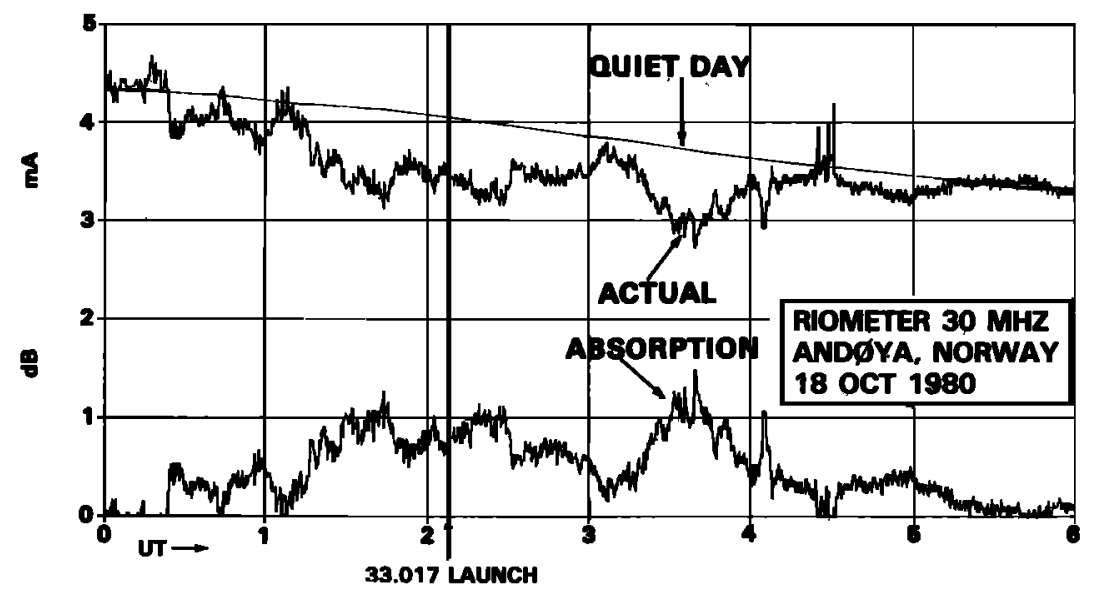

Fig. 3. Riometer absorption records for And $\phi y a$, Norway, during MAE I with the indicated launch times (UT) for (a) 30.010 and 33.015 and $(b) 33.017$. The quiet day curves along with computer-calculated absorption in decibels are also displayed.

For MAE I, we incorporated multichannel solid state detectors on all flights. These provided us with continuous data for the electron spectrum so that the assumption of a nonvarying source was no longer required. It also permitted us to compare spectral distributions acquired by the two techniques as shown in the next section of this paper. This section discusses the details of the techniques used to analyze the data and produce energy deposition curves.

\section{$X$ Ray Analysis}

Except for the high-energy integral channels on each $X$ ray detector, all $\mathrm{X}$ ray data were prepared in differential energy bins of 5-10, 10-20, 20-40, and, for MAE II, 40-80 keV. For those detectors operating with integral channels, this was accomplished through subtraction of count rates between adjacent energy channels. All data used in the analysis were taken below heights (normally about $70 \mathrm{~km}$ ) containing significant contamination by direct particle penetration or locally produced bremsstrahlung. These were also corrected for background cosmic rays by subtracting values obtained from the quiet night flights in Alaska (15.165) and Norway (30.011,
33.016). Such background contributions are normally small in comparison with the fluxes encountered on disturbed nights at all altitudes above the stratosphere. The data were converted to absolute fluxes making use of the instrument geometric factors.

The $X$ ray fluxes so derived were then used to obtain the energy deposition profiles using techniques already described in detail by Barcus et al. [1981]. Briefly, at any given altitude, the fluxes measured in situ were used to generate a source function at the top of the atmosphere by correcting for atmospheric absorption upward from the measurement height. The energy spectral shape was constructed by comparing the fluxes in each of the $X$ ray detector channels. Once the source function was derived, it was used to reconstruct the in situ measurement. The derived source function was considered correct when by a series of iterations, the original measured fluxes and those reconstructed by the source function were found to compare within satisfactory limits.

An exponential shape was specified for the source function in order to conduct the above procedures. It was also assumed that the source height existed at $100 \mathrm{~km}$. The actual pro- 
a

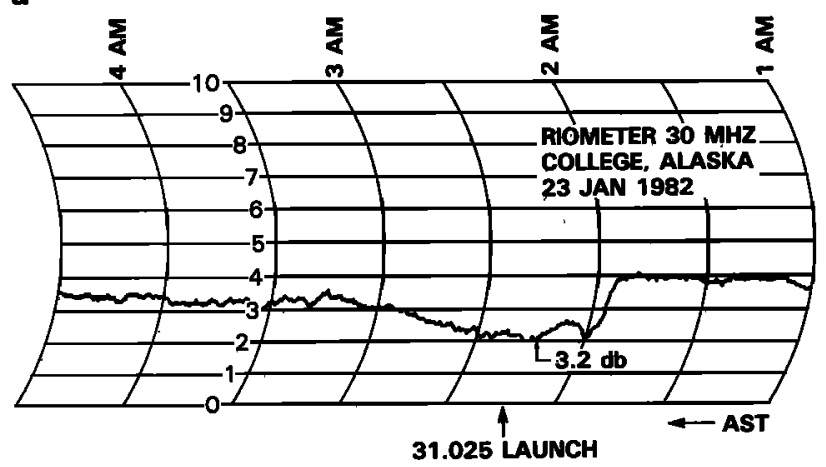

b

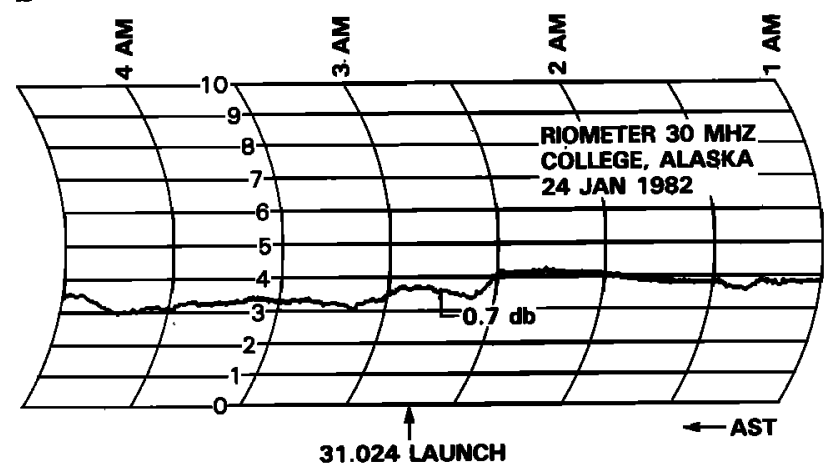

Fig. 4. Riometer absorption records for College, Alaska, during MAE II with the indicated launch times (AST) for $(a) 31.025$ and $(b)$ 31.024 .

duction layer extends from about 90 - to $120-\mathrm{km}$ altitude, but $100 \mathrm{~km}$ is the approximate altitude of maximum ionization resulting from the $X$ ray producing parent electrons and therefore a reasonable approximation for the source height.

\section{Relativistic Electron Analysis}

The in situ measurement of electron flux was provided by one of the three instruments described in the first part of this section. The details of our treatment in obtaining electron spectra are presented in the appendix. Here we summarize some of the features of the treatment to maintain continuity within the text: At the top of the atmosphere the distribution was fit to an exponential shape of the form $F=F_{0}$ exp $\left(-E / E_{0}\right)$, where $F$ and $F_{0}$ represent directional flux, $E$ is energy, and $E_{0}$ is the folding energy. For some of the flights (see the appendix), on-board electron spectrometers provided measured values of $F_{0}$ and $E_{0}$ directly. For those cases limited by the integral flux measurements from the $X$ ray or Geiger tube detectors, we determined that $F$ had to be broken into several intervals with varying values for $E_{0}$ and $F_{0}$ to obtain a best fit between the derived source flux at the top of the atmosphere and the in situ measurements at various heights within the absorption region of the atmosphere. This was accomplished by integrating through the atmosphere in a series of iterations which were continued until satisfactory agreement was obtained between the derived and measured flux. Table 4 lists the parameters obtained for all relativistic electron distributions measured during the four rocket programs reported in this work. As an example, Figure 6 depicts the spectra representing the derived parameters in Table 4 for the flights during Aurorozone I and II.

The charged particle data from detectors flown in MAE I provided electron spectra with at least 4-ms resolution within the atmosphere. It was therefore possible to construct a source spectral distribution at the top of the atmosphere for any period of interest. The spectra derived for MAE I are presented in the next section along with their derived energy deposition profiles.

\section{Results AND Discussion}

Beginning with our first crude detection of relativistic electrons from the contamination of the $X$ ray detectors flown during Aurorozone' I [Goldberg, 1979; Barcus et al., 1980], we compare the energy deposition curves for the various events studied within the scope of this work. Greatest emphasis and detail are concentrated on the results of MAE I in And $\phi y a$, Norway, since the details of the relativistic electron spectral distributions could be examined more accurately with the use of solid state detectors. These have permitted comparison of spectral distributions obtained with the time-averaged energy range results from $X$ ray or Geiger tube detector data with those acquired instantaneously from the solid state detectors. Furthermore, for the case of NASA 30.010 and 33.015, which were in the air simultaneously, we have obtained comparisons of the temporal structure for a single event from corresponding data sets at high and low altitudes. The comparisons made during MAE I have enhanced our ability to evaluate the characteristics of the events observed in Alaska during MAE II.

\section{Aurorozone I}

Figure 7 depicts the energy deposition profile curves obtained during upleg (cf. Figure 5) for the two reported events during Aurorozone I. They exhibit characteristics which typify all of the energy deposition curves to be reported here for the various events studied. The energy sources can be divided into distinct altitude domains governed by the nature of the penetrating radiation. The measured fluxes consist of the bremsstrahlung $X$ rays $(>5 \mathrm{keV})$ and the electrons $(0.015$ to 1.0 $\mathrm{MeV}$ ). The $\mathrm{X}$ ray fluxes, which have normally been treated as the primary nighttime ionizing source from about 40 to 80 $\mathrm{km}$, are found here to dominate only between about $40 \mathrm{~km}$ and $60 \mathrm{~km}$. Below about $40 \mathrm{~km}$ the galactic cosmic ray background is the most important source. However, from $60 \mathrm{~km}$ to $80 \mathrm{~km}$, relativistic electrons dominate as the primary radiation responsible for nighttime ionization. Above $80 \mathrm{~km}$ we show the calculated energy deposition from the measured parent energetic electrons responsible for the bremsstrahlung $X$ rays. This could be determined on these flights (and only on these flights) because each carried a multiple array of Geiger tubes to high altitude.

As noted earlier, each of the measurements occurred during an auroral breakup period. From the riometer records in Figure 1, it is apparent that each event extended north to Fort Yukon, although during the launch period on September 21, 1976, the effects at Fort Yukon had mostly subsided. This is also evidenced in the electron density contours of Figure 5. The absolute absorption values indicated on each riometer curve were obtained by comparing the disturbed absorption profile to a quiet curve obtained a few days prior to or after each event. These reference curves are shown for illustration in the Aurorozone I riometer records. We find that the September 21 event exhibited significantly lower absorption $(\sim 2 \mathrm{~dB})$ than the event on September $23(\sim 4 \mathrm{~dB})$, and during each launch period, both events had begun to recover. 

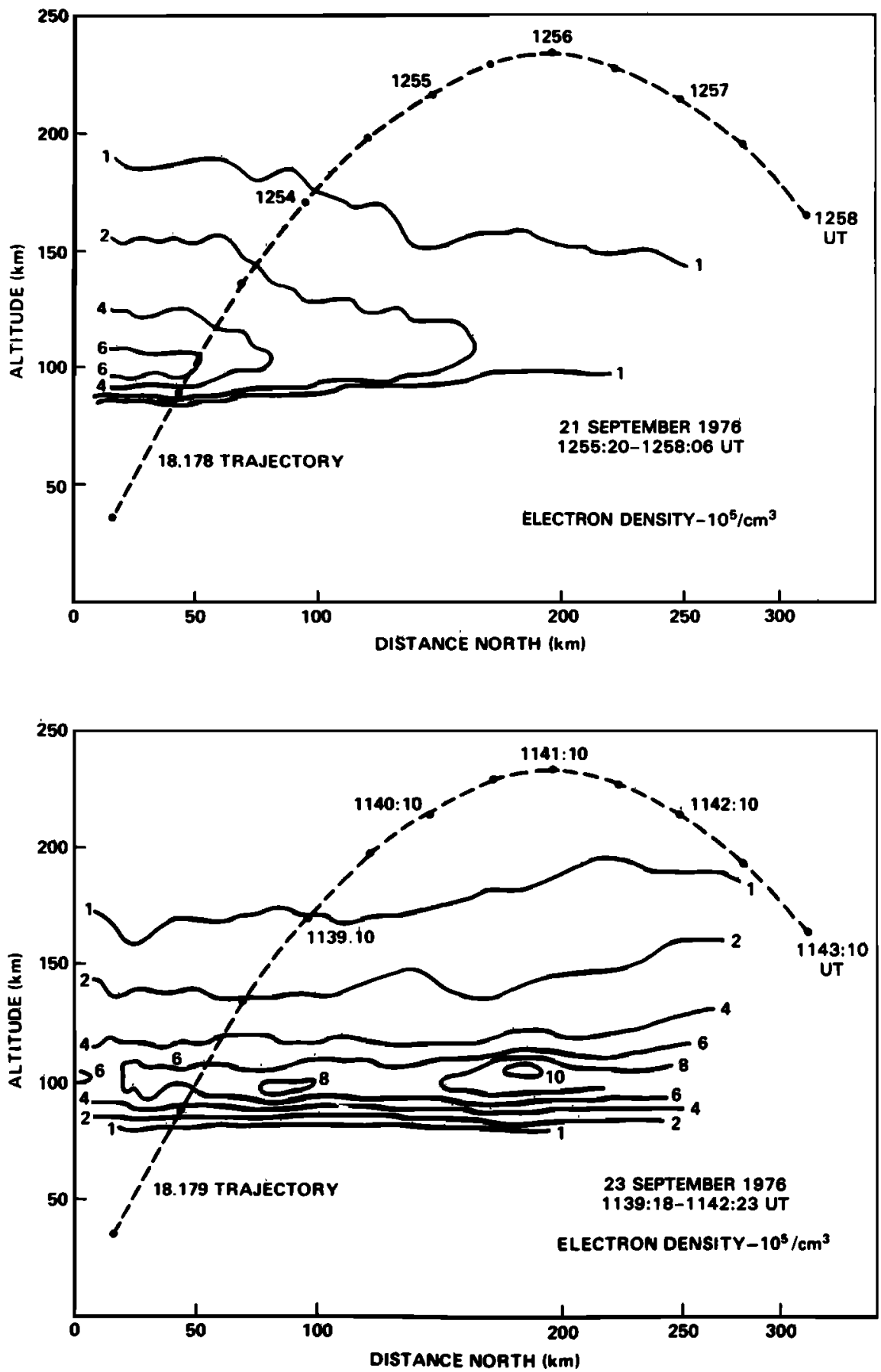

Fig. 5. Electron density contour maps (Chatanika) and superimposed trajectories for Nike Tomahawk rockets 18.178 and 18.179 during Aurorozone $\mathbf{I}$.

Comparison of the energy deposition curves for the two events (Figure 7) shows that the $\mathrm{X}$ ray component was significantly more intense and spectrally, harder on September 23 (18.179). This is consistent with the increased riometer absorption seen during this event, since the main absorption must occur in the free electron region, which is created primarily by parent electrons stopped above $80 \mathrm{~km}$. The relativistic electron energy flux is similar for the two events, with that on September 23 exhibiting more intense and spectrally hard characteristics. The hardness for these energy sources can be judged by the penetration depth of the energy deposition within the atmosphere (cf. Figure 8).

\section{Aurorozone II}

During Aurorozone II, 15.169 was launched into an auroral breakup exhibiting the highest riometer absorption ( $5.5 \mathrm{~dB}$; cf. Figure 2) of the events studied here. The energy deposition curves for this flight are illustrated in Figure 9. Because of the limited apogee attained by the Super Arcas rockets used for the XRG payload flights in Aurorozone II, these data were 
TABLE 3. Instrument Characteristics

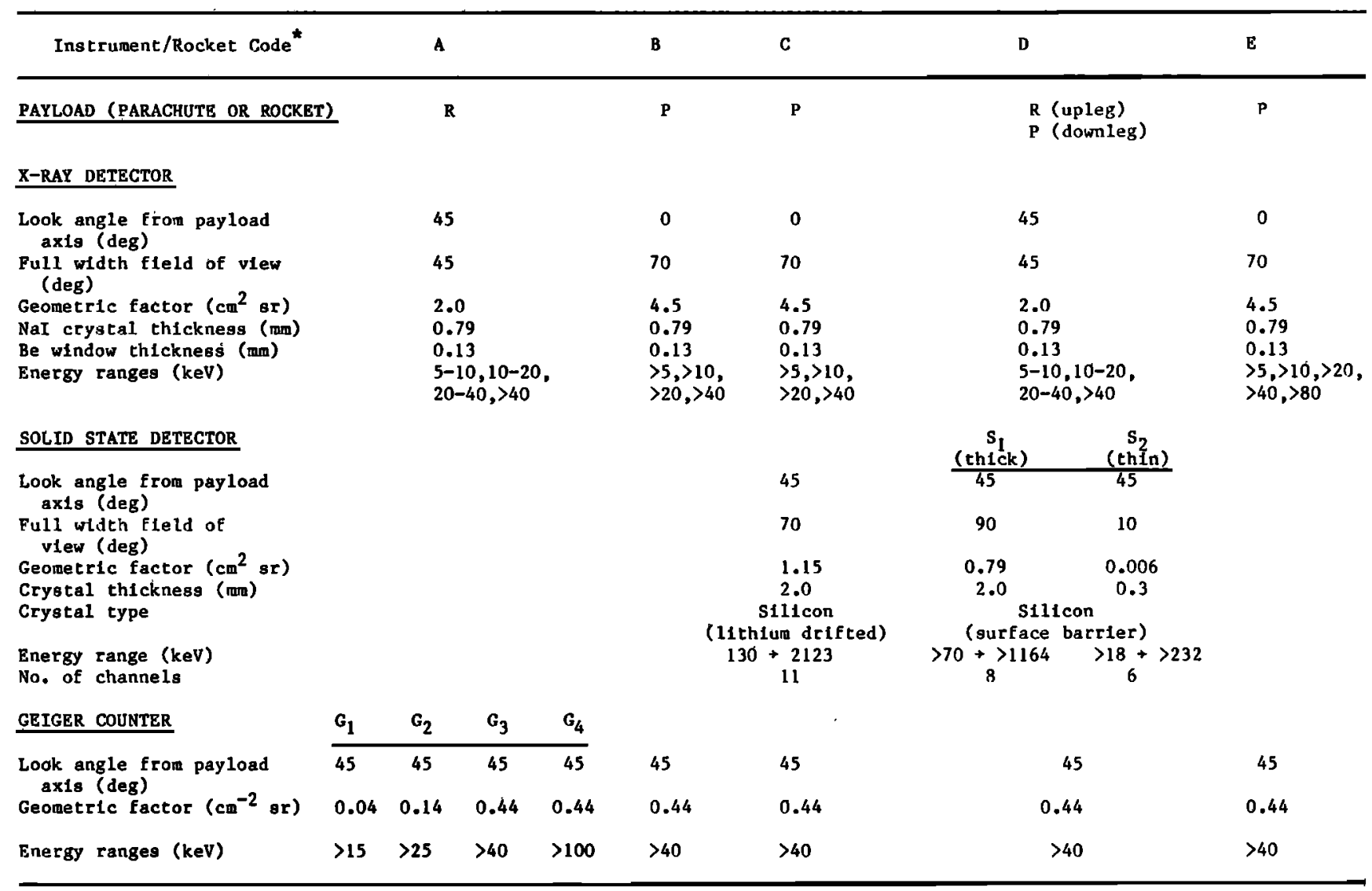

* See Table 1.

obtained only to altitudes between 60 and $70 \mathrm{~km}$. Comparison of these curves with those presented for Aurorozone I show that for this event, the $\mathrm{X}$ ray flux exhibited a harder spectrum causing significant energy deposition to altitudes below $40 \mathrm{~km}$. This implies that a more energetic parent electron distribution created higher-electron production within the primary riometer absorption region between $80 \mathrm{~km}$ and $100 \mathrm{~km}$ (not shown). For this event the relativistic electron flux showed a spectrum similar to that of 18.178 between $0.1 \mathrm{MeV}$ and $1.0 \mathrm{MeV}$ but also contained a high-energy component up to $2.5 \mathrm{MeV}$.

Also shown in Figure 9 are the energy depósition curves obtained during the flight of 15.168 on March 29, 1978. These results along with those of 31.024 in MAE II (discussed later in this section) are the most unusual of any reported here because they indicate a period when relativistic electrons were the exclusive energy source of consequence in the mesosphere. The observed weak contribution from $X$ rays is consistent with the low absorption ( $1.2 \mathrm{~dB})$ measured by the riometer for this period and indicates small fluxes for the softer electrons. The relativistic electron fluxes observed here above $1.0 \mathrm{MeV}$ (below about $57 \mathrm{~km}$ ) are the most intense and spectrally hard of all events studied in this work, with a high-energy component detectable out to $3.0 \mathrm{MeV}$. This is most easily seen by comparing the height where the relativistic electron energy deposition crossover with that of galactic cosmic rays occurs. For 15.168 the crossover was at an unusually low altitude $(\sim 45 \mathrm{~km}$ ), indicating the presence of $2.5-\mathrm{MeV}$ electrons ( $\mathrm{c}$. Figure 8).

\section{MAE I}

MAE I was conducted in And $\phi y a$, Norway, during October 1980. The two active periods selected for study during this program were determined to be aurorally active but relatively stable in space and time, as would be expected during conditions of diffuse aurorae with or without pulsations. Both events on October 7 and 18 met these constraints. The And $\phi$ ya riometer records shown in Figure 3 depict both the actual trace (milliamperes) and the calculated absorption (decibels) for each night studied. The times of launch are indicated by a vertical line. For October 7 we launched into the recovery phase of an event which reached a maximum absorption of $2 \mathrm{~dB}$. For October 18 the riometer records indicate a relatively weak absorption event near $1 \mathrm{~dB}$, which continued for a period approaching 2 hours. This period better met the characteristics of slowly fluctuating pulsations, but at a low flux level.

For this study we have concentrated on the October 7 event, which was measured with two near-simultaneous ( $1 \mathrm{~min}$ apart) rocket flights carrying an array of complementary instrumentation. Figure 10 is a sample of the measured count rates for the 5- to $10-\mathrm{keV}$ energy losses in the $\mathrm{X}$ ray detector from both 30.010 and 33.015. The abscissa for 30.010 has been offset $60 \mathrm{~s}$ to permit coincidence of the measured data in real time. We note that at the two heights where the trajectories coincide, the count rates nearly match as expected. The large fluctuations in the $\mathbf{3 0 . 0 1 0}$ data near $160 \mathrm{~s}$ correspond to large 
payload pitch oscillations during the initial phase of parachute deployment. The rapid decay of the count rates with decreasing altitude on 30.010 is attributed to expected peak absorption of 5- to $10-\mathrm{keV} X$ rays between $75 \mathrm{~km}$ and $50 \mathrm{~km}$. This absorption can also be seen in the data of the more rapidly falling 33.015 payload within the same altitude range. The large count rates observed on 33.015 above $80-\mathrm{km}$ altitude are caused by electron contamination rather than atmospheric bremsstrahlung $X$ rays.

A similar plot is presented in Figure 11 for energy losses greater than $40 \mathrm{keV}$. Here, nearly all counts acquired above approximately $75-\mathrm{km}$ altitude are those due to relativistic electrons. Hence the high-altitude data of 33.015 show the time variations for the integral relativistic electron distribution $(>150 \mathrm{keV}$ ). The decay in count rates observed between 60 $\mathrm{km}$ and $80 \mathrm{~km}$ is that caused by atmospheric absorption of the relativistic electrons, since $X$ rays of energy $>40 \mathrm{keV}$ are absorbed at much lower altitudes $(\sim 35 \mathrm{~km})$. The plot also indicates that pulsations in the electron flux were occurring every 1 to $2 \mathrm{~min}$.

Figure 12 displays the solid state detector data from $\mathbf{3 0 . 0 1 0}$ in integral form ( $>131 \mathrm{keV}$ ). Following payload stabilization near $170 \mathrm{~s}$, we observe a series of oscillations with much higher frequency than seen on the time-compressed plot of $\mathrm{X}$ ray data in Figure 11. The arrows mark two times where analyses of the electron spectra have been performed for the purpose of the following discussion. The arrow near $176 \mathrm{~s}$ marks a period considered to represent the background relativistic electron count rates during the event in progress. The arrow near $170 \mathrm{~s}$ marks either the occurrence of a particle burst within the event or a spatial variation for the incoming flux. The fact that the burst near $170 \mathrm{~s}$ was observed on the high-altitude flight (33.015) simultaneously indicates that this

TABLE 4. Parameters Used for the Derived Differential Electron Energy Spectra

\begin{tabular}{|c|c|c|c|}
\hline $\begin{array}{c}\text { Rocket } \\
\text { Payload }\end{array}$ & $\underset{\mathrm{keV}}{\text { Energy Range, }}$ & $F_{0}$ & $\begin{array}{c}E_{0} \\
\mathrm{keV}\end{array}$ \\
\hline \multirow[t]{4}{*}{18.178} & $15-86$ & $1.2(+8)^{*}$ & 6.5 \\
\hline & $86-320$ & $7.0(+2)$ & 70 \\
\hline & $320-550$ & $1.8(+2)$ & 100 \\
\hline & $550-1000$ & $3.8(+1)$ & 150 \\
\hline \multirow[t]{3}{*}{18.179} & $15-69$ & $1.5(+8)$ & 5 \\
\hline & $69-800$ & $2.5(+2)$ & 150 \\
\hline & $800-1500$ & $1.2(+1)$ & 350 \\
\hline \multirow[t]{5}{*}{15.168} & $200-300$ & $1.9(+4)$ & 32 \\
\hline & $300-1000$ & $2.7(+0)$ & 570 \\
\hline & $1000-1600$ & $5.1(+0)$ & 420 \\
\hline & $1600-2600$ & $7.6(+0)$ & 380 \\
\hline & $2600-3500$ & $2.4(-2)$ & 2400 \\
\hline \multirow[t]{3}{*}{15.169} & $200-300$ & $8.7(+5)$ & 22 \\
\hline & $300-1300$ & $2.2(+0)$ & 270 \\
\hline & $1300-2400$ & $1.2(-1)$ & 680 \\
\hline 30.010 & $100-1000$ & $2.7(+2)$ & 60 \\
\hline \multirow{2}{*}{ and } & $100-1000$ & $1.5(+2)$ & 80 \\
\hline & $100-1000$ & $9.6(+1)$ & 100 \\
\hline \multirow[t]{2}{*}{33.017} & $150-400$ & $2.0(+4)$ & 84 \\
\hline & $400-1000$ & $8.5(+2)$ & 250 \\
\hline \multirow[t]{3}{*}{31.024} & $150-400$ & $3.2(+3)$ & 120 \\
\hline & $400-800$ & $4.2(+2)$ & 470 \\
\hline & $800-1400$ & $1.1(+2)$ & 2300 \\
\hline $\begin{array}{l}31.025 \\
\quad \text { Maximum }\end{array}$ & $150-900$ & $1.0(+5)$ & 25 \\
\hline 31.025 & $150-900$ & $1.3(+4)$ & 25 \\
\hline Minimum & & & \\
\hline
\end{tabular}

$*^{*}$ Read $1.2(+8)$ as $1.2 \times 10^{8}$.

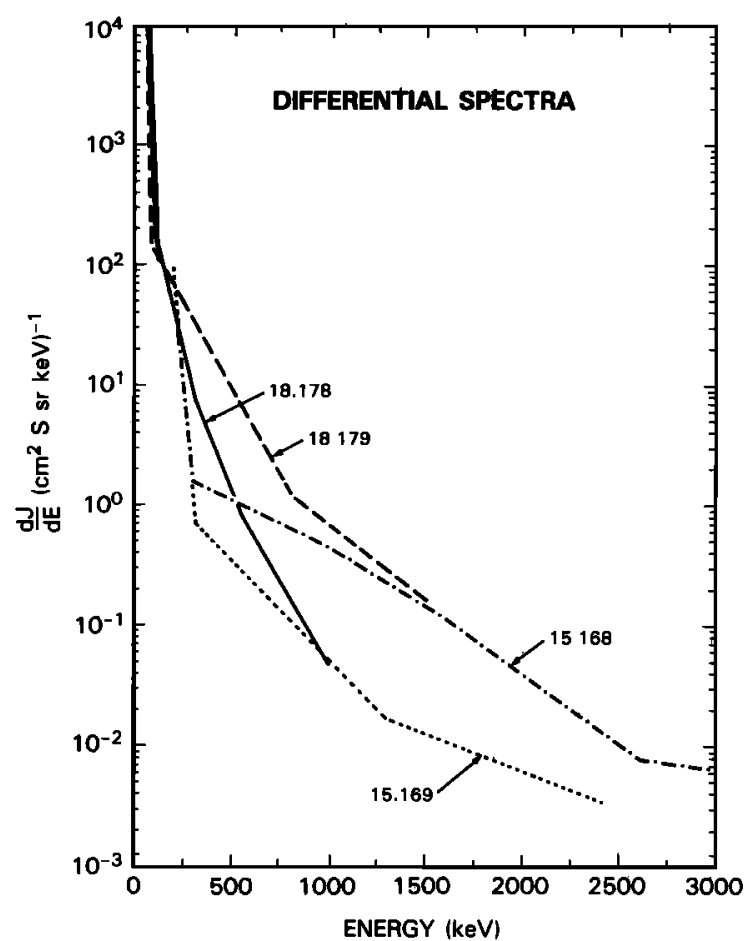

Fig. 6. Differential spectra calculated for the relativistic electron distributions measured during Aurorozone I and II. The spectra were obtained from parameters listed in Table 2.

peak in the flux was probably of temporal rather than spatial origin.

Figure 13 presents differential electron spectra which have been prepared from the data for both periods under the assumption that they can be approximated by an exponential distribution of the form $J=J_{0} \exp \left(-E / E_{0}\right)$. A series of five
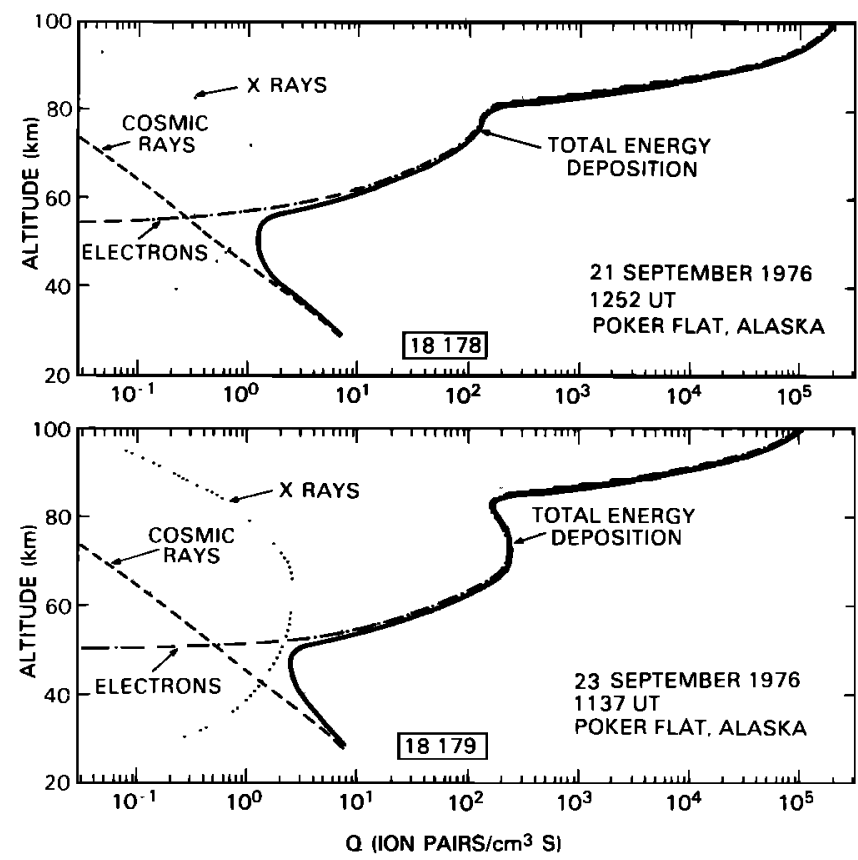

Fig. 7. Energy deposition profiles obtained during Aurorozone I from rockets 18.178 and 18.179 . The $X$ ray, electron $(>15 \mathrm{keV})$, cosmic ray background, and combined total energy deposition curves are presented in terms of ion pair production rates. 


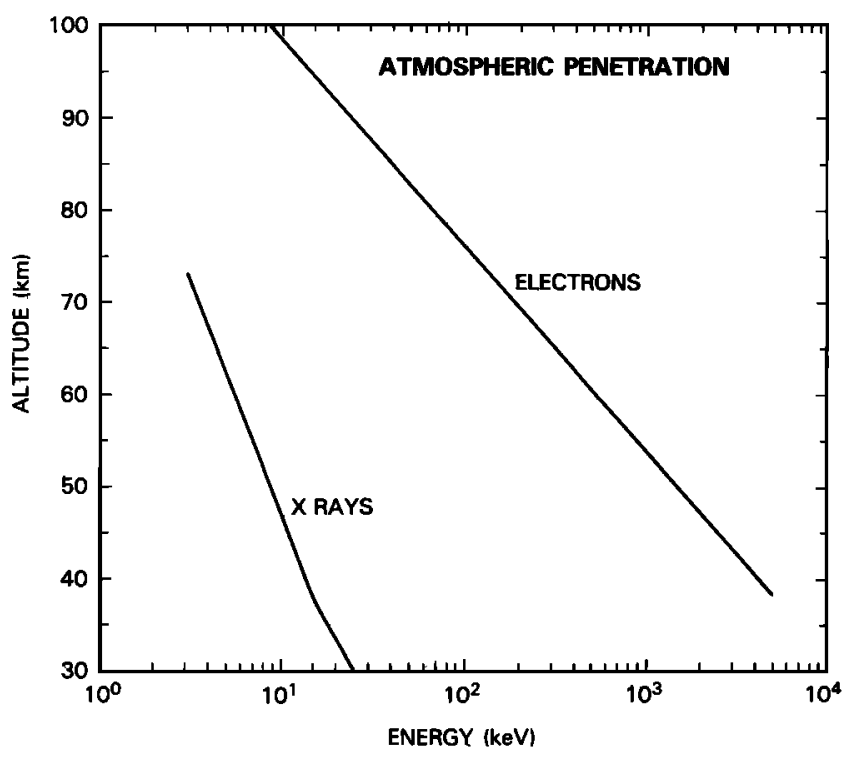

Fig. 8. Characteristic atmospheric penetration for an isotropic distribution of $X$ rays and energetic electrons. The depths represent the height by which at least $98 \%$ of the energy has been deposited.

heavy crossbar points mark the solid state data from 30.010 at $176 \mathrm{~s}$. The vertical error bar signifies one standard deviation uncertainty for the count rates obtained. The horizontal bar signifies the energy range used to specily the count rate measurement. In some cases, multiple detector energy channels were grouped together to improve the statistical reliability of the values. Three separate lines with folding energies $\left(E_{0}\right)$ of 65,80 , and $90 \mathrm{keV}$ have been drawn through the data points to indicate the range limits of the distribution. Also shown is a dashed step function carve representing the spectrum obtained from the electron data acquired in the highenergy channel of the $\mathrm{X}$ ray detector. The consistency between the two results obtained here helps justify the use of the cruder techniques already described for the determination of particle spectra and energy deposition curves acquired in the Aurorozone I and II data sets. The burst seen near $170 \mathrm{~s}$ provided the shown spectrum from the $\mathbf{3 0 . 0 1 0}$ data with a best fit for $E_{0}$ at the nominal $80-\mathrm{keV}$ value. The solid state detector aboard the high-altitude rocket 33.015 obtained electron count rate data with better time resolution. The spectrum acquired at the peak of the burst (170 s) for this flight is also displayed and exhibits nearly the same spectral shape $\left(E_{0}=75 \mathrm{keV}\right)$, although the flux intensity has increased by a factor of 10 . These results indicate that the burst represented a uniform increase in electron flux at all observed energies.

The data for the event on October 18 (33.017) have been analyzed using the $X$ ray detector to establish the energetic electron spectrum. Figure 13 illustrates the spectrum obtained by exponential fit with a dashed step function labeled " 33.017 $X$ ray." A linear fit provides a slope with an $E_{0}$ of $95 \mathrm{keV}$. Hence the relativistic electron distribution for this event exhibited a slightly harder spectrum but with an intensity at least 1 order of magnitude below that observed on October 7 .

The $\mathrm{X}$ ray detector data plus the electron spectra presented in Figure 13 have been used to construct the energy deposition curves displayed in Figure 14. For the October 7 event the spectrum obtained during the background period near 176 $s$ was used to construct three separate curves for the relativistic electron deposition, all with the same integral energy flux but with shapes representing spectral folding energies of 60,80 , and $100 \mathrm{keV}$ with corresponding values for $J_{0}$ of 267 , 150 , and $98\left(\mathrm{~cm}^{2} \mathrm{~s} \mathrm{sr} \mathrm{keV}\right)^{-1}$. This range, depicted in the figure by the shaded region, is meant to indicate the scope of values which could be derived from the data set. Here the curve labeled with a folding energy of $80 \mathrm{keV}$ is considered nominal. On these curves and on those for October 18, the profiles show a maximum near $80 \mathrm{~km}$ and begin to decrease with altitude. These high-altitude results are an accurate representation of the energy deposition caused by relativistic electrons but do not include the soft parent electron component responsible for the $\mathrm{X}$ rays, as was discussed for Aurorozone I and illustrated in Figure 7. This, of course, would induce a rapid growth in energy deposition above the $80-\mathrm{km}$ region. The $X$ ray data from both 33.015 and 30.010 have been compared to produce the $X$ ray energy deposition curves shown. For the October 18 event the results were obtained exclusively from the data of 33.017 .

The energy deposition results presented for MAE I in Figure 14 show the October 7 event to be significantly more intense for relativistic electrons but less intense for bremsstrahlung $\mathrm{X}$ rays than that on October 18 . In fact, for October 18 the relativistic comporient was one of the weakest of any event reported here. The October 7 event shows an intense relativistic component up to $0.7 \mathrm{MeV}$ but is devoid of the
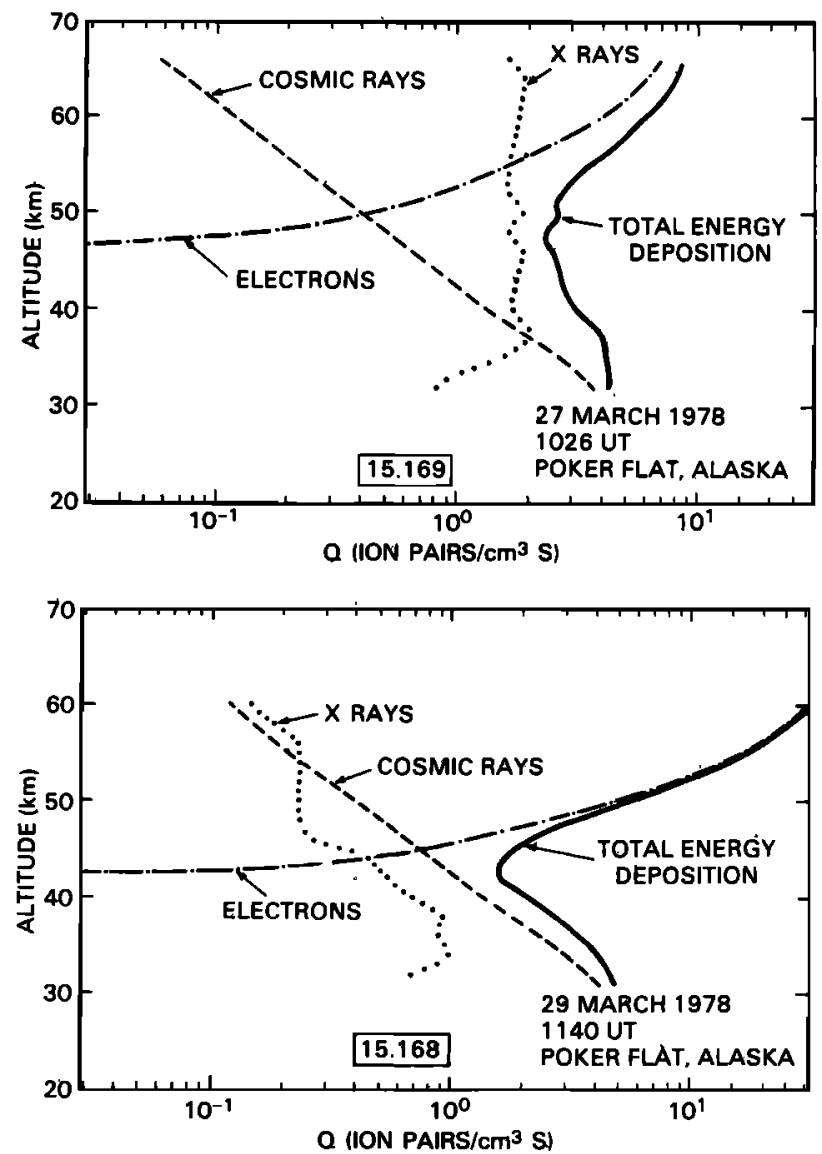

Fig. 9. Energy deposition profiles obtained during Aurorozone II from rockets 15.169 and 15.168 . The $X$ ray, relativistic electron $(>200$ $\mathrm{keV}$ ), cosmic ray background, and combined total energy deposition curves are presented in terms of ion pair production rates. The low apogee acquired for these Super Arcas flights limited data acquisition to the altitude range shown. 


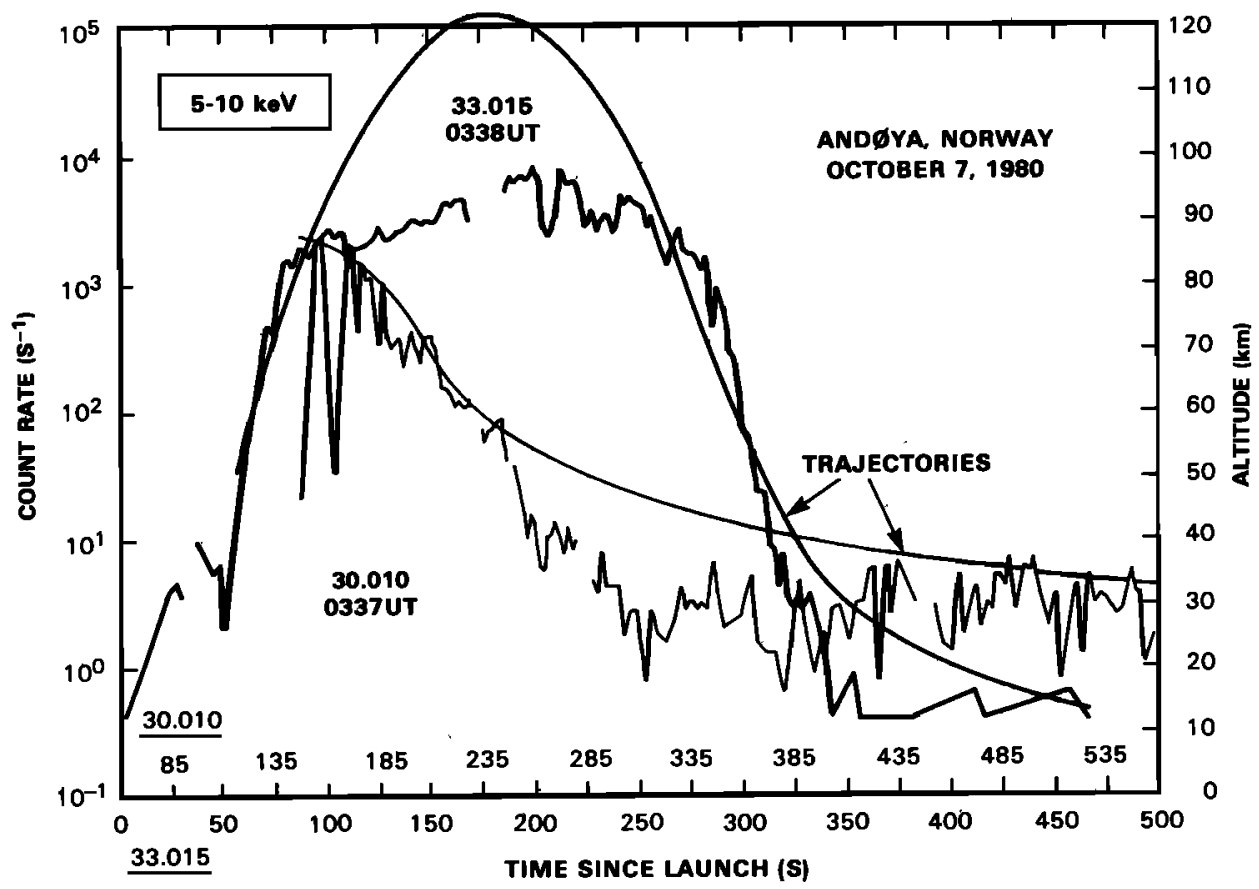

Fig. 10. $X$ ray detector count rates versus time after liftoff for the 5- to $10-\mathrm{keV}$ energy range from 33.015 and 30.010 during MAE I. The time scales for the two flights have been aligned to permit simultaneous comparison of the two data sets. The trajectory profiles for each flight (right-hand scale) are also given. Note that the count rates shown exhibit some degree of electron contamination at the upper altitudes, particularly for 33.015 .

high-energy tails commonly seen in the Alaskan data (cf. Figures 6 and 13 for a comparison of spectra). We attribute this either to statistical variations in the temporal variability associated with the occurrence of such events or to the more poleward location of And $\phi y$ a than Poker Flat within the auroral zone and the widely accepted result that the high-energy electron component diminishes with increasing magnetic latitude.

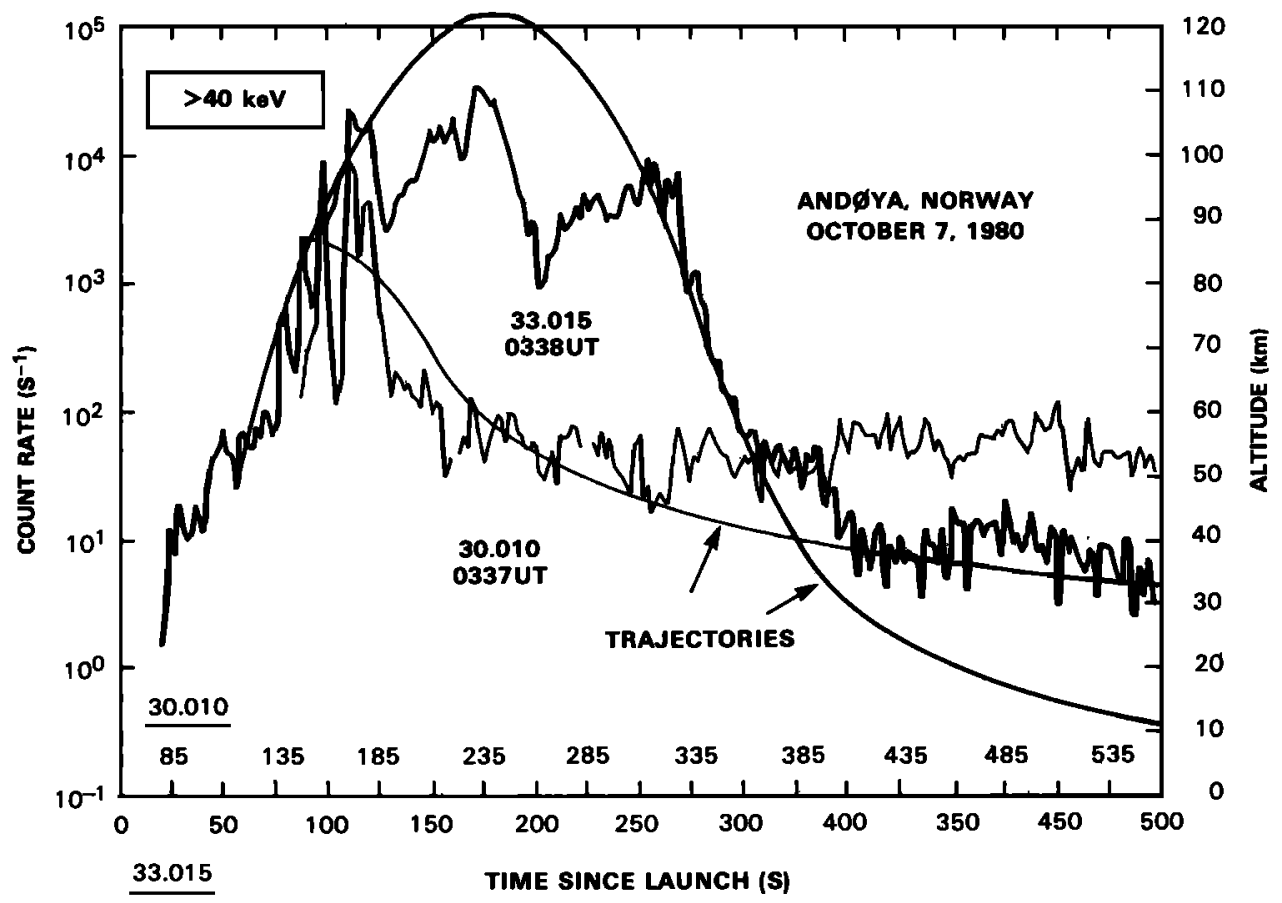

Fig. 11. $\mathrm{X}$ ray detector count rates versus time after liftofl for the $>40-\mathrm{keV}$ energy channels from 33.015 and 30.010 during MAE I. The time scales for the two flights have been aligned to permit simultaneous comparison of the two data sets. The trajectory profiles for each flight (right-hand seale) are also given. The high-altitude count rates, particularly on 33.015 above $90 \mathrm{~km}$, are almost exclusively due to energetic electrons $>150 \mathrm{keV}$ (see text), while data below $90 \mathrm{~km}$ on both flights contain electron contamination which gradually diminishes with atmospheric depth. 


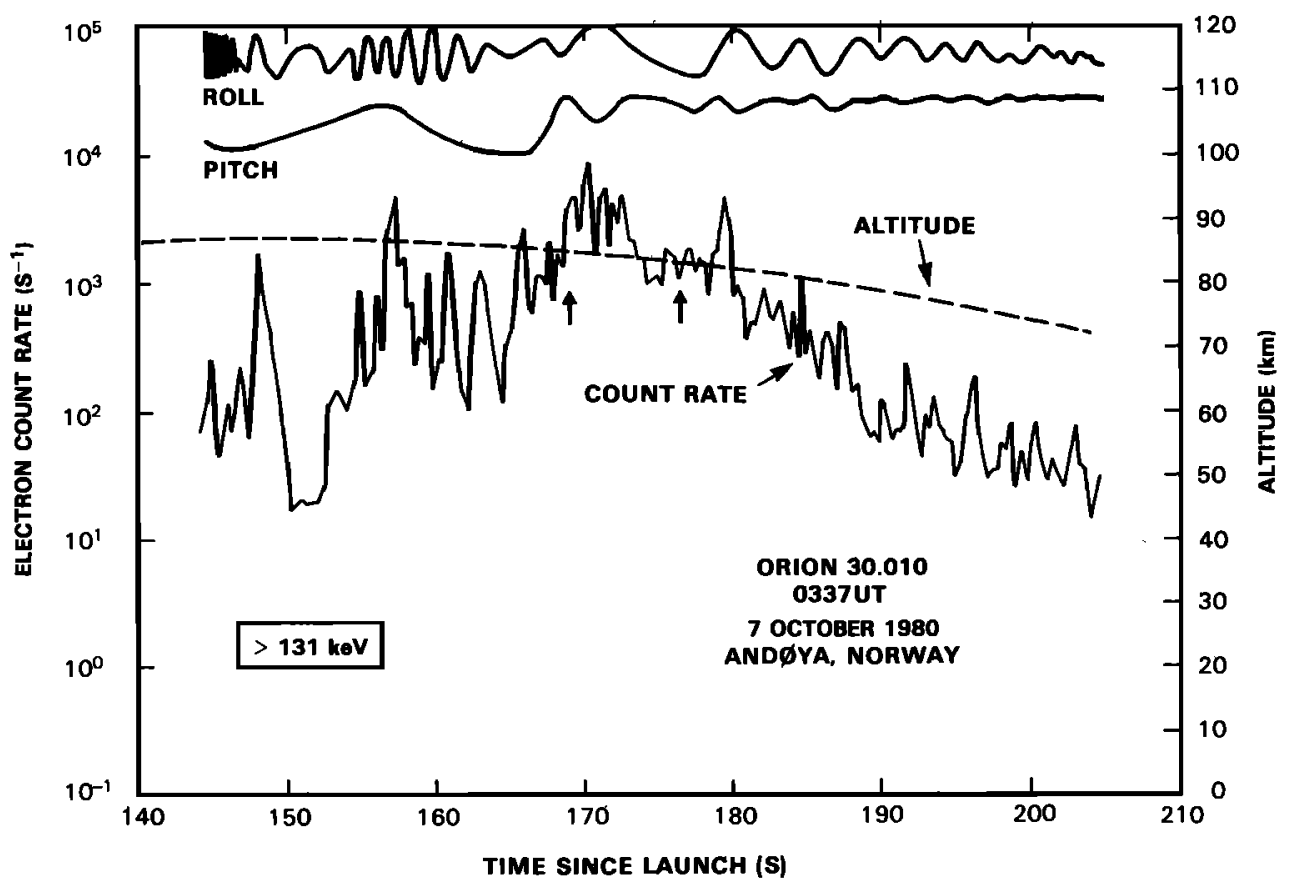

Fig. 12. The integrated count rate for relativistic electrons $(>131 \mathrm{keV})$ measured with the solid state detector on 30.010 versus time after liftoff. The simultaneous display of pitch and roll aspect data indicates intervals near parachute deployment $(\sim 150 \mathrm{~s})$ where severe modulations in aspect are reflected in the count rate data. The arrows depict burst and nonburst periods used to construct the spectra (Figure 13) for the energy deposition curves (Figure 14).

\section{$M A E I I$}

For the final results reported here, MAE II in Poker Flat, Alaska, during January 1982 , we have used the $X$ ray detector measurements for the evaluation of both the $X$ ray and relativistic electron energy deposition. Figure 15 shows the energy deposition profiles produced for each of the two events. These were calculated from spectra with characteristics for the relativistic electron distributions defined in Table 4. For the event measured on January 23 (31.025) we observed oscillations in the $\mathrm{X}$ ray data of the order of $2 \mathrm{~min}$, during which the amplitude range for count rates was about a lactor of 3 . The energy deposition profiles for these data are expressed by pairs of curves for incoming $X$ ray, relativistic electron, and total energy deposition. Once again, the anticipated large contribution to energy deposition above $70-80 \mathrm{~km}$ from parent electrons is not shown. The shaded region between the two total deposition curves marks the enhancement induced by nearly identical pulsations observed over the interval from $200 \mathrm{~s}$ to 400 s. The background energy deposition curves exhibit a weak and spectrally soft relativistic electron component but also show relatively high $\mathrm{X}$ ray fluxes dominating the cosmic ray background above $41 \mathrm{~km}$. During the pulsations the $X$ ray flux is observed to be boosted over the spectrum measured with some hardening, extending $X$ ray dominance as the primary ionization source to $33 \mathrm{~km}$. The relativistic electron flux (Table 4) is estimated to increase about a factor of 8 , although it should be noted that the absolute values determined for the relativistic electron fluxes on this flight are considered to be less accurate than those for other events reported here because of the time variations present and because the instrument threshold $(\sim 150 \mathrm{keV})$ affected measurements in the critical region of this unusually soft spectrum.

The results obtained on January 24 (31.024) were measured during stable diffuse auroral conditions with no pulsations present. The low riometer absorption $(0.7 \mathrm{~dB})$ is consistent with the absence of a strong $X$ ray component. However, the relativistic electron energy component penetrated to greater depths than that for the previous night, yielding a weaker version of the nearly pure relativistic electron event observed during Aurorozone II (15.168). The extreme differences between the energy deposition curves for the two events studied during MAE II, and only 1 day apart, emphasize the wide variability in radiation sources for each event.

\section{CONCLUSIONS}

We have presented measurements of energy deposition within the middle atmosphere during eight distinct nighttime auroral events. For each of the events studied, we have determined that relativistic electron fluxes occurred as a regular feature. This contributed an ionizing radiation component to the mesosphere significantly larger than the bremsstrahlung $X$ ray component produced by the softer parent electrons which bombard the atmosphere near $100 \mathrm{~km}$. For five of six cases in Alaska and for one of two cases in Norway, this component exceeded the $X$ ray flux by 1 or 2 orders of magnitude everywhere above $60 \mathrm{~km}$, which would lead to large enhancements in charge density and ion conductivity. The correspondence of these enhancements with the local ion chemistry and middle atmospheric electrodynamics is the topic of other work in preparation. Within the limited opportunities of this study, we have attempted to show the frequent occurrence and general characteristics of this important radiation component.

We typically observed relativistic electron energy deposition peaking between $60 \mathrm{~km}$ and $80 \mathrm{~km}$, with the Alaskan data exhibiting high-energy components above $1 \mathrm{MeV}$ which were not present in the data from Norway. The intensity of the 


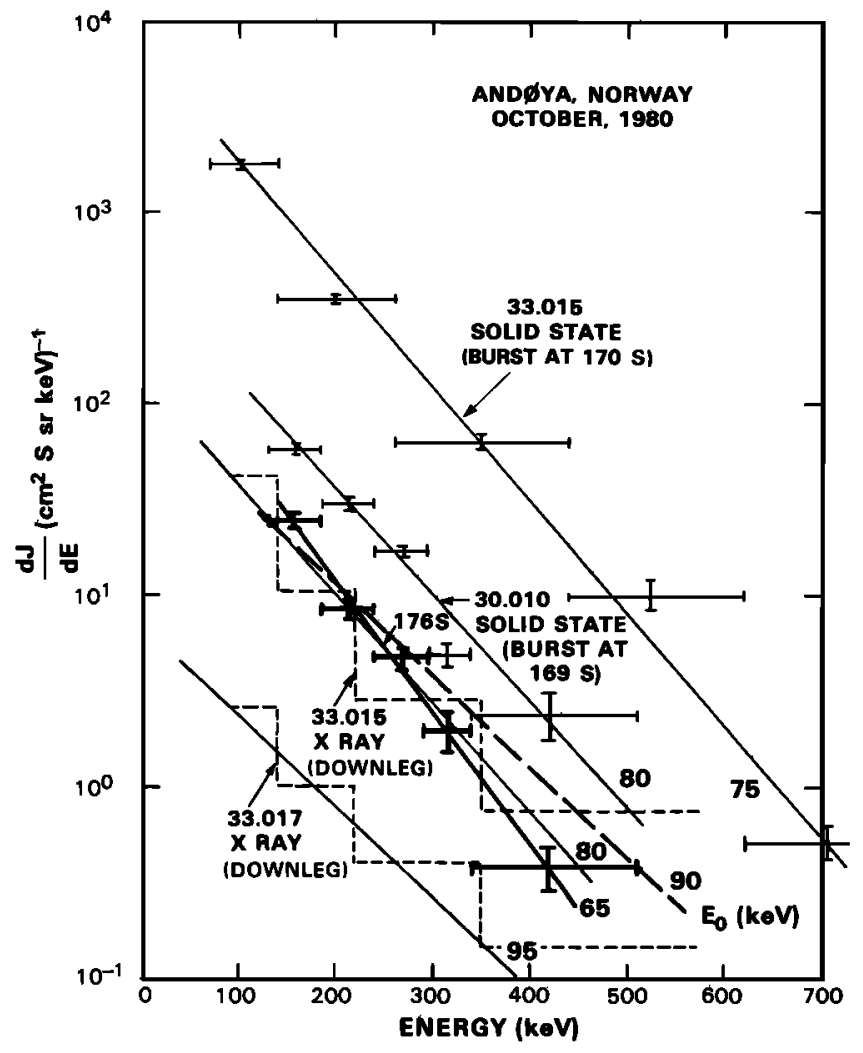

Fig. 13. Differential relativistic spectra for various detectors flown during MAE I. The solid state detector points have horizontal bars indicating each energy range and vertical bars representing one standard deviation. Different slopes with the indicated folding energies mark the limits for each measurement. Also shown are spectra obtained from the $>40-\mathrm{keV}$ channel of each $\mathrm{X}$ ray detector on 33.015 and 33.017. These are acquired by downleg averaging of the data, as opposed to instantaneous determination by the solid state detectors.

Alaskan fluxes was usually 1 to 2 orders of magnitude greater than that observed for the Norwegian fluxes. These features may be related to temporal differences between the measurements at the two sites, but they are also consistent with earlier balloon and riometer findings [e.g., Bailey and Pomerantz, 1965; Hartz and Brice, 1967; Bailey, 1968; Rosenberg et al., 1972; Thorne, 1974; Larsen and Thomas, 1974] which have implied that REP distributions soften toward the poleward side of the auroral zone.

We note that relativistic electrons were observed to precipitate in quantity for every auroral breakup or postbreakup condition studied. The only variables among events were the hardness and intensity of the energy spectrum. Also, since two of the Alaskan measurements of relativistic electrons (15.168, March 29, 1978; 31.024, January 24, 1982) occurred during a nonbreakup period when little activity was indicated from riometer or magnetometer (not shown) records, one is left to infer that such nighttime REP's could occur more frequently than is normally thought, as suggested by Thorne [1980] and Andreoli [1980]. The possible importance of such undetected events is further emphasized by our determination that the March 29 REP was the most energetic of all events studied in this work.

The Alaskan event of January 23, 1982, allowed us the rare opportunity to compare energy deposition enhancements induced by long-period auroral pulsations. This event was found to exhibit increases for both the measured $X$ rays and relativistic electrons. The $X$ ray spectrum, particularly during a pulsation period, was one of the hardest of any studied within these reported results.

Finally, we note that peak ion production rates in the mesosphere from relativistic electrons reached values of 100 ion pairs $/ \mathrm{cm}^{3} \mathrm{~s}$ in the mesosphere between $60 \mathrm{~km}$ and $80 \mathrm{~km}$. Comparison with several of the recently studied larger solar proton events [e.g., Crutzen et al., 1975; Reagan et al., 1978; McPeters et al., 1981; Solomon et al., 1983] shows that for these larger events, the energy deposition in the same altitude region is at least 1 to 2 orders of magnitude greater than the REP deposition reported here. However, the integrated effect of REP events may more than compensate for their reduced energy contribution. This would be true provided the modifications induced by these radiation sources cause effects with lifetimes significantly longer than the interval between events.

\section{APPENDIX}

\section{Determination of Electron Spectra}

Direct measurements. Wherever possible, the electron spectrum used in the energy deposition calculations has been obtained directly from on-board particle spectrometers. Those flights and the corresponding energy ranges are the following: 18.178 and $18.179,15-150 \mathrm{keV} ; 33.015$ and $33.017,30-100$
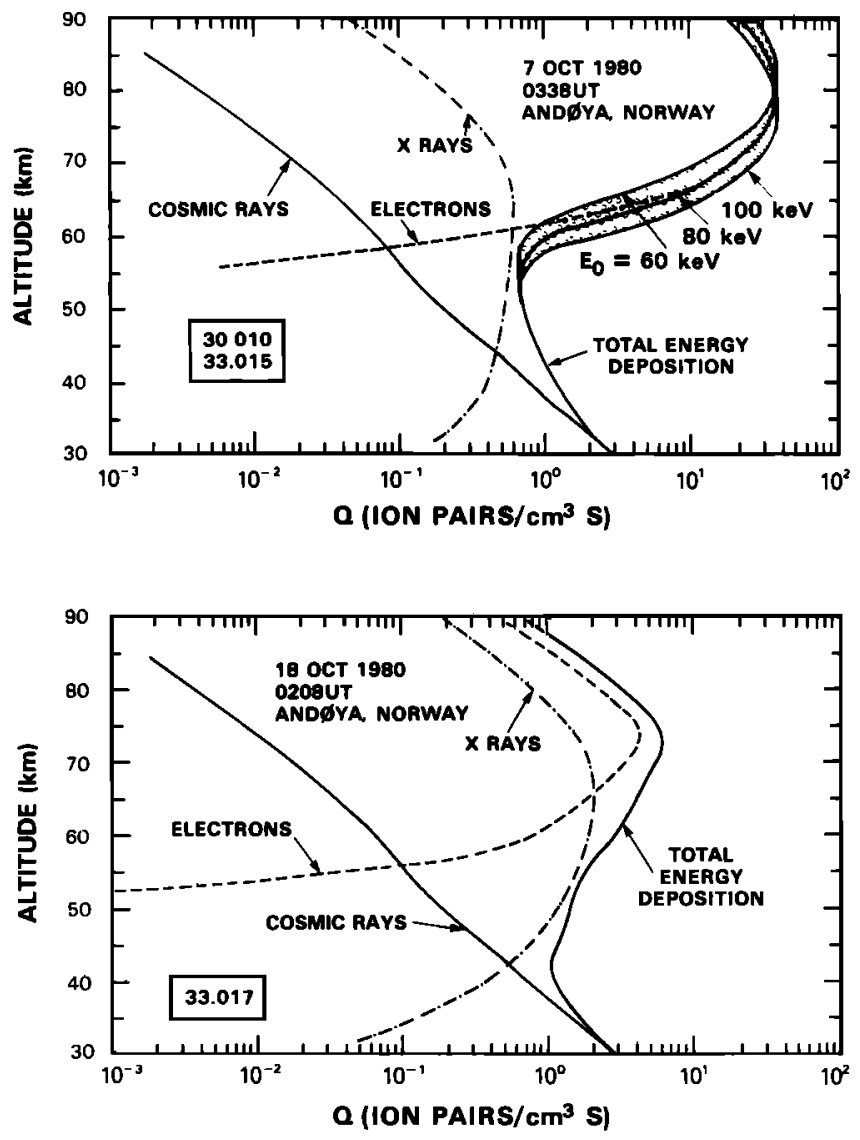

Fig. 14. Energy deposition profiles obtained during MAE I from rockets $30.010,33.015$, and 33.017 . The $X$ ray, relativistic electron $(>100 \mathrm{keV})$, cosmic ray background, and combined total energy deposition curves are presented in terms of ion pair production rates. The range of values for relativistic electron deposition brackets the range of folding energies depicted in Figure 13 for the nonburst period (176s). 

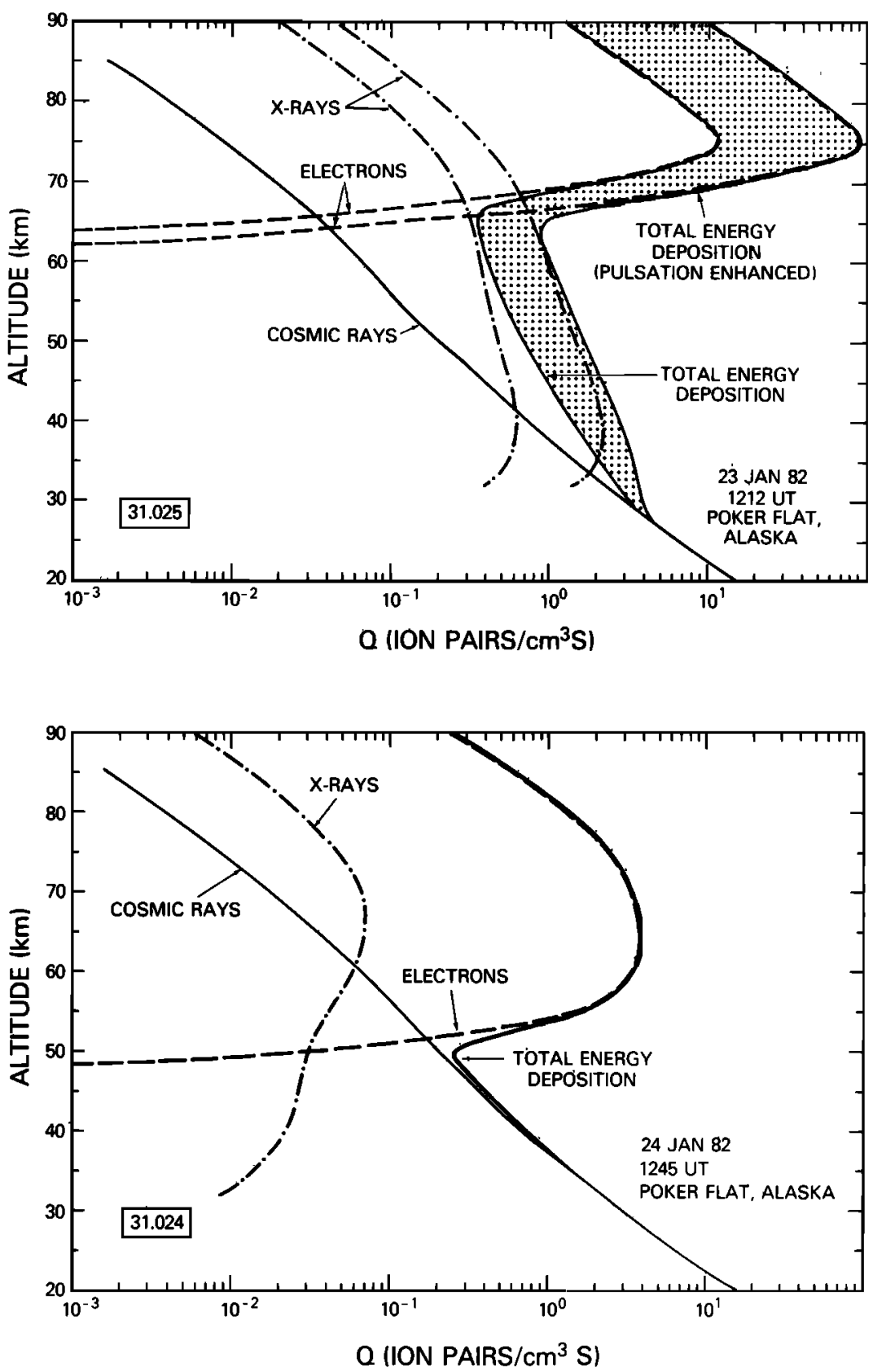

Fig. 15. Energy deposition profiles oblained during MAE II from rockets 31.025 and 31.024 . The $X$ ray, relativistic electron ( $>150 \mathrm{keV}$ ), cosmic ray background, and combined total energy deposition curves are presented in terms of ion pair production rates. For 31.025 the electron and $X$ ray curves are given for both background and pulsation conditions. The pulsation enhancement in total energy deposition is shown by the shaded region.

$\mathrm{keV} ; 30.010,100-1000 \mathrm{keV}$. Otherwise, the spectrum has been deduced from the atmospheric absorption of the integral electron flux as measured by single counters and/or by electron contamination of the $\mathrm{X}$ ray scintillation detector, as described in the next part of this section.

Indirect measurements. Using either the response of the $\mathrm{X}$ ray detector or the Geiger counter, the integral electron flux,

$$
F_{\text {obs }}\left(z, E>E_{\mathrm{min}}\right)\left(\mathrm{cm}^{2} \mathrm{~s} \mathrm{sr}\right)^{-1}
$$

was obtained as a function of altitude. The subscript "obs" refers to the fact that this was an observed electron flux. The minimum energies, $E_{\min }$, detected by the Geiger counter and $\mathrm{X}$ ray detector were $40 \mathrm{keV}$ and $150 \mathrm{keV}$, respectively. Our ob- jective was to translate this altitude dependent electron flux into the differential electron flux at the top of the atmosphere,

$$
\frac{d F}{d E}\left(z_{\mathrm{top}}, E\right)\left(\mathrm{cm}^{2} \mathrm{~s} \mathrm{sr} \mathrm{keV}\right)^{-1}
$$

where $E>E_{\min }$, using range-energy considerations.

We assumed for the purposes of this study that the electron spectra could be represented by a series of exponential distributions with different intensities and $e$-folding energies, depending on the electron energy range. A single pair of parameters, $F_{0}$ and $E_{0}$, was found insufficient to reliably represent the electron spectra over the entire range. Thus the energy range 
was divided into several bands with a pair of parameters determined for each band. The form is given by

$$
F=F_{0} \exp \left(-E / E_{0}\right)
$$

The rocket data in most cases only extend up to $65 \mathrm{~km}$. Using the range-energy relation given in equation (A3) of the next section of this appendix, we found that the primary electrons must have energies of $>250 \mathrm{keV}$ in order to reach $65 \mathrm{~km}$.

The procedure used in deriving $d F / d E(z, E)$ was the following: A reasonable estimate of $d F / d E\left(z_{\text {lop }}, E\right)$ was initially made. As the spectrum of electrons, $d F / d E\left(z_{\text {top }}, E\right)$ impinged on the atmosphere, the electrons lost energy to the atmosphere in a manner outlined in the next section of this appendix. A spectrum at each altitude, $d F / d E(z, E)$, was calculated and integrated over the energy variable to give the result, $F_{\text {cal }}\left(z, E>E_{\min }\right)$, the subscript "cal" referring to the fact that this electron spectrum was calculated.

The $F_{\mathrm{cal}}\left(z, E>E_{\mathrm{min}}\right)$ was then compared with the $F_{\text {obs }}(z$, $E>E_{\text {min }}$ ) to obtain an agreement within $25 \%$ at all altitudes, at which time the iteration process was terminated. If agreement was not within these limits, then a new iteration was required, adjusting $d F / d E\left(z_{\text {top }}, E\right)$ until the requirement was met. The $d F / d E\left(z_{\text {top }}, E\right)$ from the last iteration was used to calculate the energy input into the middle atmosphere by electrons using the method given in the next section of this appendix.

\section{Determination of Electron Energy Deposition}

In order to compute the energy loss at an altitude $z$, we use

$$
\begin{aligned}
D(z)= & {\left[\rho(z) \int_{E_{1}(z)}^{E_{2}(z)} \int_{0}^{2 \pi} \int_{0}^{\pi / 2} \cos \theta \frac{d F}{d E}(z, E)\right.} \\
& \cdot G(E, \theta) d \Omega d E] \cdot[\Delta M(z)]^{-1}\left(\mathrm{keV} \mathrm{cm}^{-3} \mathrm{~s}^{-1}\right)
\end{aligned}
$$

$D(z)$ is the energy loss at altitude $z, d F / d E(z, E)\left(\mathrm{cm}^{2} \mathrm{~s}\right.$ sr $\mathrm{keV})^{-1}$ is the differential electron spectrum in energy $E$, polar angle $\theta$, and azimuthal angle $\phi$ at altitude $z . E_{2}(z)$ is the maximum energy to which the calculations were carried. $E_{1}(z)$ is equal to $E_{\min }$ at the top of the atmosphere but can be less than $E_{\min }$ as the electrons lose their energy to the atmosphere. This spectrum is assumed to be isotropic at $z_{\text {ipp }}$, the top of the atmosphere, which is $150 \mathrm{~km}$ in our model.

$$
G(E, \theta)=E-\left[-\frac{\Delta M(z)}{A} \sec \theta+E^{B}\right]^{1 / B}(\mathrm{keV})
$$

is the energy loss in the atmospheric column of length $\Delta M(z)$ by an electron of energy $E$ traveling in the direction of polar angle $\theta$. Parameters $A$ and $B$ come from the equation for the electron range,

$$
R(E)=A\left(\frac{E}{1000 \mathrm{keV}}\right)^{B}\left(\mathrm{~g} \mathrm{~cm}^{-2}\right)
$$

The parameters $A$ and $B$ were found by fitting the electron mean range-energy table given by Berger and Seltzer [1972]. Using $A=0.864$ and $B=1.73$ for electron energies from 1 $\mathrm{keV}$ to $295 \mathrm{keV}$ and $A=0.295$ and $B=1.23$ for energies from $295 \mathrm{keV}$ to $3500 \mathrm{keV}$ gave range values within $3 \%$ of Berger and Seltzer. Here, $\Delta M(z)\left(\mathrm{g} \mathrm{cm}^{-2}\right)$ is the atmospheric mass in a column $2 \mathrm{~km}$ high centered on altitude $z, \rho(z)\left(\mathrm{g} \mathrm{cm}^{-3}\right)$ is the atmospheric density at altitude $z$, and $E(z)(\mathrm{keV})$ is the electron energy at the top of the column $\Delta M(z)$.

The technique used to deposit the energy of an electron spectrum in the atmosphere was the following: The atmosphere (U.S. Standard Atmosphere, 1976) was divided into 75 segments each $2 \mathrm{~km}$ in width. The isotropic undegraded downward electron spectrum $d F / d E\left(z_{\text {top }}, E\right)$ was input into the top of the atmosphere at 35 angular intervals spaced evenly between an incident polar angle of $0 \mathrm{rad}$ and $\pi / 2 \mathrm{rad}$. In the first altitude slab, $z_{\text {top }}$ replaced $z$. In each subsequent loweraltitude slab the atmospheric column and volume densities were updated to be consistent with the new altitude, and the electron energy $E$ was corrected for the energy loss, $G(E, \theta)$, in the next higher-altitude slab. Such a procedure was followed to the lower altitudes until the ground was reached.

Acknowledgments. This research was made possible through the dedicated and highly professional efforts of Sounding Rocket personnel at the Goddard Space Flight Center. We also thank Joe Modlin, who was payload instrument engineer on all flights and contributed greatly to the success of each program. Finally, we thank $L$. Gesell, K. Brown, P. Twigg, and A. Reid for their competent assistance in various aspects of the data analysis.

The Editor thanks J. B. Reagan and another referee for their assistance in evaluating this paper.

\section{REFERENCES}

Andreoli, L. J., Relativistic electron precipitation: An observational study, Ph.D. dissertation, Univ. of Calif., Los Angeles, 1980.

Bailey, D. K., Some quantitative aspects of electron precipitation in and near the auroral zone, Rev. Geophys. Space Phys., 6, 289-346, 1968.

Bailey, D. K., and M. A. Pomerantz, Relativistic electron precipitation into the mesosphere at subauroral latitudes, J. Geophys. Res., $70,5823-5830,1965$.

Barcus, J. R., R. A. Goldberg, E. R. Hilsenrath, and J. D. Mitchell, Middle atmosphere response to measured relativistic electrons, in Collection of Extended Abstracts Presented at ICMUA Sessions and IUGG Sympasium 18, Commission on Meteorology of the Upper Atmosphere, National Center for Atmospheric Research, Boulder, Colo., 1980.

Baircus, J. R., R. A. Goldberg, and L. H. Gesell, X-ray scanning of overhead aurorae from rockets, J. Atmos. Terr. Phys., 43, 10031013,1981 .

Berger, M. J, and S. M. Seltzer, Bremsstrahlung in the atmosphere, $J$. Atmos. Terr. Phys., 34, 85-108, 1972.

Crutzen, P. J., I. S. A. Isáksen, and G. C. Reid, Solar proton events: Stratospheric sources of nitric oxide, Science, 189, 457-458, 1975.

Frederick, J. E., Solar corpuscular emission and neutral chemistry in the earth's middle atmosphere, $J$. Geophys. Res., 81, 3179-3186, 1976.

Goldberg, R. A., An experimental search for causal mechanisms in sun/weather-climatic relationships, in Solar Terrestrial Infuences on Weather and Climate, edited by B. M. McCormac and T. A. Seliga, D. Reidel, Hingham, Mass., 1979.

Goldberg, R. A., J. R. Barcus, L. A. Treinish, and R. R. Vondrak, Mapping of auroral $\mathrm{X}$ rays from rocket overflights, J. Geophys. Res., 87, 2509-2524, 1982.

Hartz, T. R., and N. M. Brice, The general pattern of auroral particle precipitation, Planet. Space Sci., I5, 301-329, 1967.

Heath, D. F., A. J. Krueger, and P. J. Crutzen, Solar proton event: Influence on strảtospheric ozone, Science, 197, 886-889, 1977.

Larsen, T. R., and G. R. Thomas, Energy spectra measured during a relativistic electron precipitation event on 2 February 1969, $J$. Atmos. Terr. Phys., 36, 1613-1622, 1974.

Larsen, T. R., J. B. Reagan, W. L. Imhol, J. E. Montbriand, and J. S. Belrose, A coordinated study of energetic electron precipitation and $D$ region electron concentrations over Ottawa during disturbed conditions, J. Geophys. Res., 81, 2200-2212, 1976.

McPeters, R. D., C. H. Jackman, and E. G. Stassinopoulos, Observations of ozone depletion associated with solar proton events, $J$. Geophys. Res., 86, 12,071-12,081, 1981.

Nicolet, M., On the production of nitric oxide by cosmic rays in the mesosphere and stratosphere, Planet. Space Sci., 23, 637-649, 1975.

Porter, H. S., C. H. Jackman, and A. E. S. Green, Efficiencies for production of atemic nitrogen and oxygen by relativistic proton impact in air, J. Chem. Phys., 65, 154-167, 1976. 
Reagan, J. B., Ionization processes, in Dynamical and Chemical Coupling of Neutral and Ionized Atmospheres, edited by B. Grandal and J. A. Holtet, p. 145, D. Reidel, Hingham, Mass., 1977.

Reagan, J. B., R. C. Gunton, J. E. Evans, R. W. Nightingale, R. G Johnson, W. L. Imhof, and R. E. Meyerott, Effects of the August 1972 solar particle events on stratospheric ozone, Rep. LMSCD630455, Lockheed Palo Alto Res. Lab., Palo Alto, Calif., Oct. 1978.

Reagan, J. B., R. E. Meyerott, R. W. Nightingale, R. C. Gunton, R. G. Johnson, J. E. Evans, W. L. Imhof, D. F. Heath, and A. J. Krueger, Effects of the August 1972 solar particle events on stratospheric ozone, J. Geophys. Res., 86, 1473-1494, 1981.

Rosenberg, T. J., L. J. Lanzerotti, D. K. Bailey, and J. D. Pierson, Energy spectra in relativistic electron precipitation events, J. Atmos. Terr. Phys., 34, 1977-1990, 1972.

Solomon, S., and P. J. Crutzen, Analysis of the August 1972 solar proton event including chlorine chemistry, J. Geophys. Res., 86, 1140-1146, 1981.

Solomon, S., G. C. Reid, D. W. Rusch, and R. J. Thomas, Mesospheric ozone depletion during the solar proton event of July 13, 1982, II, Comparison between theory and measurements, Geophys. Res. Lett., 10, 257-260, 1983.

Swider, W., and T. J. Keneshea, Decrease of ozone and atomic oxygen in the lower mesosphere during a PCA event, Planet. Space Sci., 21, $1969-1973,1973$.

Thorne, R. M., A possible cause of dayside relativistic electron precipitation events, J. Atmos. Terr. Phys., 36, 635-645, 1974.

Thorne, R. M., Energetic radiation belt electron precipitation: A natural depletion mechanism for stratospheric ozone, Science, 195, 287-289, 1977.

Thorne, R. M., The potential role of relativistic electron precipitation as a natural destruction mechanism for middle atmospheric ozone, in Proceedings of the Joint IAGA/IAM AP Assembly in Seattle, National Center for Atmospheric Research, Boulder, Colo, 1978.

Thorne, R. M., The importance of energetic particle precipitation on the chemical composition of the middle atmosphere, Pure Appl. Geophys., 118, 128-151, 1980.

Thorne, R. M., and T. R. Larsen, An investigation of relativistic electron precipitation events and their association with magnetospheric substorm activity, J. Geophys. Res., 81, 5501-5506, 1976.

Vampola, A. L., Electron pitch angle scattering in the outer zone during magnetically disturbed times, J. Geophys. Res., 76, 4685$4688,1971$.

Vampola, A. L., and D. J. Gorney, Electron energy deposition in the middle atmosphere, J. Geophys. Res., 88, 6267-6274, 1983.

Weeks, L. H., R. S. CuiKay, and J. R. Corbin, Ozone measurements In the mesosphere during the solar proton event of November 2 , 1969, J. Atmos. Sci., 29, 1138-1142, 1972.

J. R. Barcus, Department of Physics, University of Denver, Denver, CO 80208

R. A. Goldberg, Laboratory for Planetary Atmospheres, NASA Goddard Space Flight Center, Code 961, Greenbelt, MD 20771.

C. H. Jackman, Laboratory for Planetary Atmospheres, NASA Goddard Space Flight Center, Code 964, Greenbelt, MD 20771.

F. S $\phi$ raas, Department of Physics, University of Bergen, Bergen, Norway.

(Received October 17, 1983;

revised February 20, 1984; accepted March 30, 1984.) 